\title{
THE RADIAL VELOCITY EXPERIMENT (RAVE): FIRST DATA RELEASE
}

\author{
M. Steinmetz, ${ }^{1}$ T. Zwitter, ${ }^{2}$ A. Siebert, ${ }^{1,3}$ F. G. Watson, ${ }^{4}$ K. C. Freeman, ${ }^{5}$ U. Munari, ${ }^{6}$ R. Campbell, ${ }^{7}$ M. Williams, ${ }^{5}$ \\ G. M. Seabroke, ${ }^{8}$ R. F. G. Wyse, ${ }^{9}$ Q. A. Parker,${ }^{4,7}$ O. Bienaymé, ${ }^{10}$ S. Roeser, ${ }^{11}$ B. K. Gibson, ${ }^{12}$ G. Gilmore, ${ }^{8}$ \\ E. K. Grebel ${ }^{13}$ A. Helmi, ${ }^{14}$ J. F. Navarro, ${ }^{15}$ D. Burton, ${ }^{4}$ C. J. P. Cass, ${ }^{4}$ J. A. Dawe, ${ }^{4,16}$ K. Fiegert, ${ }^{4}$ \\ M. Hartley, ${ }^{4}$ K. S. Russell ${ }^{4}$ W. Saunders,${ }^{4}$ H. Enke, ${ }^{1}$ J. Bailin, ${ }^{17}$ J. Binney, ${ }^{18}$ J. Bland-Hawthorn, ${ }^{4}$ \\ C. Boeche, ${ }^{1}$ W. Dehnen, ${ }^{19}$ D. J. Eisenstein, ${ }^{3}$ N. W. Evans, ${ }^{8}$ M. Fiorucci, ${ }^{6}$ J. P. Fulbright, ${ }^{11}$ \\ O. Gerhard, ${ }^{13}$ U. Jauregi, ${ }^{2}$ A. Kelz, ${ }^{1}$ L. Mijović, ${ }^{2}$ I. Minchev, ${ }^{20}$ G. Parmentier,${ }^{8}$ \\ J. Peñarrubia, ${ }^{15}$ A. C. Quillen, ${ }^{19}$ M. A. Read, ${ }^{21}$ G. Ruchti, ${ }^{11}$ R.-D. Scholz, ${ }^{1}$ \\ A. Siviero, ${ }^{6}$ M. C. Smith, ${ }^{14}$ R. Sordo, ${ }^{6}$ L. Veltz, ${ }^{10}$ S. Vidrih, ${ }^{8}$ \\ R. von Berlepsch, ${ }^{1}$ B. J. Boyle, ${ }^{22}$ and E. Schilbach ${ }^{11}$ \\ Received 2006 April 13; accepted 2006 June 1
}

\begin{abstract}
We present the first data release of the Radial Velocity Experiment (RAVE), an ambitious spectroscopic survey to measure radial velocities and stellar atmosphere parameters (temperature, metallicity, and surface gravity) of up to one million stars using the Six Degree Field multiobject spectrograph on the $1.2 \mathrm{~m}$ UK Schmidt Telescope of the Anglo-Australian Observatory. The RAVE program started in 2003, obtaining medium-resolution spectra (median $R=7500)$ in the Ca-triplet region (8410-8795 $\AA$ ) for southern hemisphere stars drawn from the Tycho- 2 and SuperCOSMOS catalogs, in the magnitude range $9<I<12$. The first data release is described in this paper and contains radial velocities for 24,748 individual stars $(25,274$ measurements when including reobservations). Those data were obtained on 67 nights between 2003 April 11 and 2004 April 3. The total sky coverage within this data release is $\sim 4760 \mathrm{deg}^{2}$. The average signal-to-noise ratio of the observed spectra is 29.5 , and $80 \%$ of the radial velocities have uncertainties better than $3.4 \mathrm{~km} \mathrm{~s}^{-1}$. Combining internal errors and zero-point errors, the mode is found to be $2 \mathrm{~km} \mathrm{~s}^{-1}$. Repeat observations are used to assess the stability of our radial velocity solution, resulting in a variance of $2.8 \mathrm{~km} \mathrm{~s}^{-1}$. We demonstrate that the radial velocities derived for the first data set do not show any systematic trend with color or signal-to-noise ratio. The RAVE radial velocities are complemented in the data release with proper motions from Starnet 2.0, Tycho-2, and SuperCOSMOS, in addition to photometric data from the major optical and infrared catalogs ( Tycho-2, USNO-B, DENIS, and the Two Micron All Sky Survey). The data release can be accessed via the RAVE Web site.
\end{abstract}

Key words: catalogs — stars: fundamental parameters — surveys

Online material: color figures

\section{INTRODUCTION}

Within the past decade it has been increasingly recognized that many of the clues to the fundamental problem of galaxy formation in the early universe are contained in the motions and chemical composition of long-lived stars in our Milky Way galaxy (see, e.g., Freeman \& Bland-Hawthorn 2002). The recent dis-

\footnotetext{
${ }^{1}$ Astrophysikalisches Institut Potsdam, An der Sterwarte 16, D-14482 Potsdam, Germany.

${ }^{2}$ Department of Physics, University of Ljubljana, Jadranska 19, Ljubljana, Slovenia.

3 Steward Observatory, University of Arizona, 933 North Cherry Avenue, Tucson, AZ 85287-0065.

4 Anglo-Australian Observatory, P.O. Box 296, Epping, NSW 1710, Australia.

5 Research School of Astronomy and Astrophysics, Mount Stromlo Observatory, Cotter Road, Weston Creek, Canberra, ACT 72611, Australia.

6 INAF Osservatorio Astronomico di Padova, Via dell'Osservatorio 8, Asiago I-36012, Italy.

7 Macquarie University, Sydney, NSW 2109, Australia.

8 Institute of Astronomy, University of Cambridge, Madingley Road, Cambridge CB3 0HA, UK.

9 Johns Hopkins University, 366 Bloomberg Center, 3400 North Charles Street, Baltimore, MD 21218.

${ }^{10}$ Observatoire de Strasbourg, 11 Rue de L'Université, 67000 Strasbourg, France.

11 Astronomische RechenInstitut, Moenchhofstrasse 12-14, Heidelberg D-69120, Germany.
}

covery of several instances of tidal debris in our Galaxy challenges the view laid down in the seminal paper by Eggen et al. (1962), who envision the Galaxy to be formed in one major monolithic collapse at an early epoch, followed by a period of relative quiescence lasting many gigayears. These examples include the discovery of the tidally distorted/disrupted Sagittarius dwarf galaxy (Ibata et al. 1994), the photometrically identified low-latitude Monoceros structure in the Sloan Digital Sky Survey (SDSS;

\footnotetext{
${ }^{12}$ University of Central Lancashire, Preston PR1 2HE, UK.

13 Astronomisches Institut, Universität Basel, Venusstrasse 7, Binningen $\mathrm{CH}-4102$, Switzerland

${ }^{14}$ Kapteyn Astronomical Institute, University of Groningen, Postbus 800, 9700 AV Groningen, Netherlands.

${ }^{15}$ University of Victoria, P.O. Box 3055, Station CSC, Victoria, BC V8W 3P6, Canada.

${ }^{16}$ Deceased. This paper is dedicated to the memory of John Alan Dawe (1942-2004), Astronomer-in-Charge of the UK Schmidt Telescope 1978-1984 and enthusiastic RAVE observer 2003-2004.

17 Centre for Astrophysics and Supercomputing, Swinburne University of Technology, P.O. Box 218, Hawthorn, VIC 3122, Australia.

${ }^{18}$ Rudolf Peierls Centre for Theoretical Physics, University of Oxford, 1 Keble Road, Oxford OX1 3NP, UK.

19 University of Leicester, University Road, Leicester LE1 7RH, UK.

${ }^{20}$ University of Rochester, Rochester, NY 14627-0171.

21 University of Edinburgh, Edinburgh EH9 3HJ, UK.

22 Australia Telescope National Facility, P.O. Box 76, Epping, NSW 1710, Australia.
} 
Yanny et al. 2003), and the multitude of features in higher latitude fields (Belokurov et al. 2006).

Furthermore, within the context of the concordance $\Lambda \mathrm{CDM}$ scenario, sophisticated computer simulations of structure growth within a cold dark matter (CDM) universe have now begun to shed light on how the galaxy formation process may have taken place in a hierarchical framework (see, e.g., Steinmetz \& Navarro 2002; Abadi et al. 2003; Brook et al. 2005; Governato et al. 2004; Sommer-Larsen et al. 2003). These analyses lead to a reinterpretation of structures such as the Eggen moving groups (Navarro et al. 2004) and the $\omega$ Cen globular cluster (Meza et al. 2005) in terms of merger remnants. In fact, in the extreme case, structures and old stars, in even the thin Galactic disk, are attributed to accretion events (Abadi et al. 2003; Meza et al. 2005). In addition, strong evidence for the accretion and assimilation of satellite galaxies can also be seen for other galaxies in the Local Group, in particular the "great stream" in M31 (Ibata et al. 2001). However, whether the Galaxy can indeed be formed by a sequence of merging events, as predicted by current cosmological models of galaxy formation, or whether the few well-established accretion and merging remnants - which account for only a small fraction of the stellar mass of the Galaxy - are all there is, is still a largely unanswered question. Large kinematic surveys are needed, as are large surveys that derive chemical abundances, since both kinematics signatures and elemental abundance signatures persist longer than do spatial overdensities. It is still unclear (Wyse \& Gilmore 2006) whether the observed chemical properties of the stars in the thick disk (Gilmore et al. 1995) can be brought into agreement with a scenario that sees the thick disk primarily as the result of accretion events (Abadi et al. 2003). A similar question arises owing to the distinct age distribution (Unavane et al. 1996; see, however, Abadi et al. 2006) and chemical elemental abundance distributions of stars in the stellar halo and in low-mass dwarf galaxies of the Local Group (Tolstoy et al. 2003; see, however, Robertson et al. [2005] and Bullock \& Johnston [2005]).

Stellar clusters, spiral arms, and the Galactic bar leave an imprint in the chemical and stellar velocity distribution in the solar neighborhood (Dehnen 2000; Quillen \& Minchev 2005; de Simone et al. 2004) as well. Multidimensional databases are required to investigate and differentiate between these processes and structure caused by satellite accretion.

The growing awareness of the importance of the "fossil record" in the Milky Way Galaxy in constraining galaxy formation theory is reflected by the increasing number of missions designed to unravel the formation history of the Galaxy. Stellar spectroscopy plays a crucial role in these studies, not only providing radial velocities (RVs) as a key component of the six-dimensional phase space of stellar positions and velocities but also providing much-needed information on the gravity and chemical composition of individual stars. An example of the power of such multidimensional stellar data sets has recently been shown by Helmi et al. (2006), who, by using a combination of proper motions and distances from the Hipparcos catalog (Perryman et al. 1997) and spectra from the Geneva-Copenhagen Survey (GCS; Nordström et al. 2004), were able to identify several accretion candidates within the immediate neighborhood of the Sun.

However, despite the importance of stellar spectroscopy, the past decades have seen only limited progress. Soon after Vogel (1873) measured the RVs of Sirus and Procyon, Seabroke $(1879)^{23}$ performed one of the first surveys, measuring 68 RVs for 29 stars, followed by 699 observations of 40 stars (Seabroke 1887) and 866 observations of 49 stars (Seabroke 1889). Since then,

\footnotetext{
${ }^{23}$ The great-great-grandfather of G. Seabroke, coauthor of this paper.
}

over the next $125 \mathrm{yr}, \mathrm{RVs}$ for some 50,000 stars have become available in the public databases of the Centre de Données astronomiques de Strasbourg (CDS). This is surprisingly few compared to the more than 1 million galaxy redshifts measured within the past decade. This sample of stellar RVs has recently been increased substantially by the GCS, containing RVs for 16,682 nearby dwarf stars, and by Famaey et al. (2005), who published 6691 RVs for apparently bright giant stars. Both catalogs were part of the Hipparcos follow-up campaign.

With the advent of wide field multiobject spectroscopy (MOS) fiber systems in the 1990s, pioneered particularly at the AngloAustralian Observatory (AAO) with FOCAP, AUTOFIB, and most recently with the Two Degree Field (2dF) and Six Degree Field (6dF) instruments on the Anglo-Australian Telescope (AAT) and UK Schmidt Telescope (UKST), respectively (e.g., Lewis et al. 2002; Watson et al. 2000), the possibility of undertaking wide-area surveys with hemispheric coverage became feasible. Initially, such projects were more concerned with largescale galaxy and quasar redshift surveys (e.g., Colless et al. 2001 for $2 \mathrm{dF}$; Jones et al. 2004 for $6 \mathrm{dF}$ ). Apart from the samples of several hundred to a few thousand stars obtained prior to the commissioning of AAOmega at the AAT (see, e.g., Kuijken \& Gilmore 1989a, 1989b, 1989c; Gilmore et al. 1995, 2002; Wyse \& Gilmore 1995), no large-scale, wide-area stellar spectroscopy projects had been undertaken in our own galaxy. This has now changed with new surveys such as SDSS-II SEGUE already under way, with a planned delivery of 240,000 spectra by mid-2008 (Newberg 2003), and the capabilities of AAOmega on the AAT. Over a slightly longer time frame, the Radial Velocity Experiment (RAVE), ${ }^{24}$ the survey we describe in this paper, is expected to provide spectra for up to 1 million stars by 2011. This trend for large stellar surveys will culminate with the ESA cornerstone mission Gaia, which, in addition to astrometric information, will provide multiepoch RVs for up to 100 million stars by 2018. Each of these surveys has its own unique aspect, and they are largely complementary in capabilities and target sample.

With a RV error of about $2 \mathrm{~km} \mathrm{~s}^{-1}$ and $80 \%$ of measurements better than $3.4 \mathrm{~km} \mathrm{~s}^{-1}$, the RAVE velocities are accurate enough for almost any Galactic kinematic study. RVs are, however, just one of the necessary stellar parameters: proper motions, distances, and chemical abundances are also needed. Proper motions of varying accuracy are available for most of the RAVE stars via Starnet 2.0, Tycho-2, or the SuperCOSMOS Sky Survey (SSS). Already, some of the cooler dwarfs $(J-K>0.5)$ with more accurate proper motions can be identified as dwarfs from their reduced proper motions. For these stars it is possible to estimate all six phase-space coordinates using their photometric parallaxes.

For most of these stars, there is no previous spectroscopic information, so the RAVE sample provides many scientific opportunities. Some of the science programs in progress by RAVE team members include the following:

1. Discovery of extreme-velocity stars and estimates of the local escape velocity and total mass of the Galaxy.

2. The three-dimensional velocity distribution function of the local Galactic disk.

3. Kinematics of the main stellar components of the Galaxy.

4. Characterization of the local Galactic disk potential and the structure of the disk components.

5. Substructure in the disk and halo of the Galaxy, including the Arcturus, Sagittarius, and other star streams.

6. Elemental abundances of high-velocity stars.

\footnotetext{
${ }^{24}$ See http://www.rave-survey.org.
} 


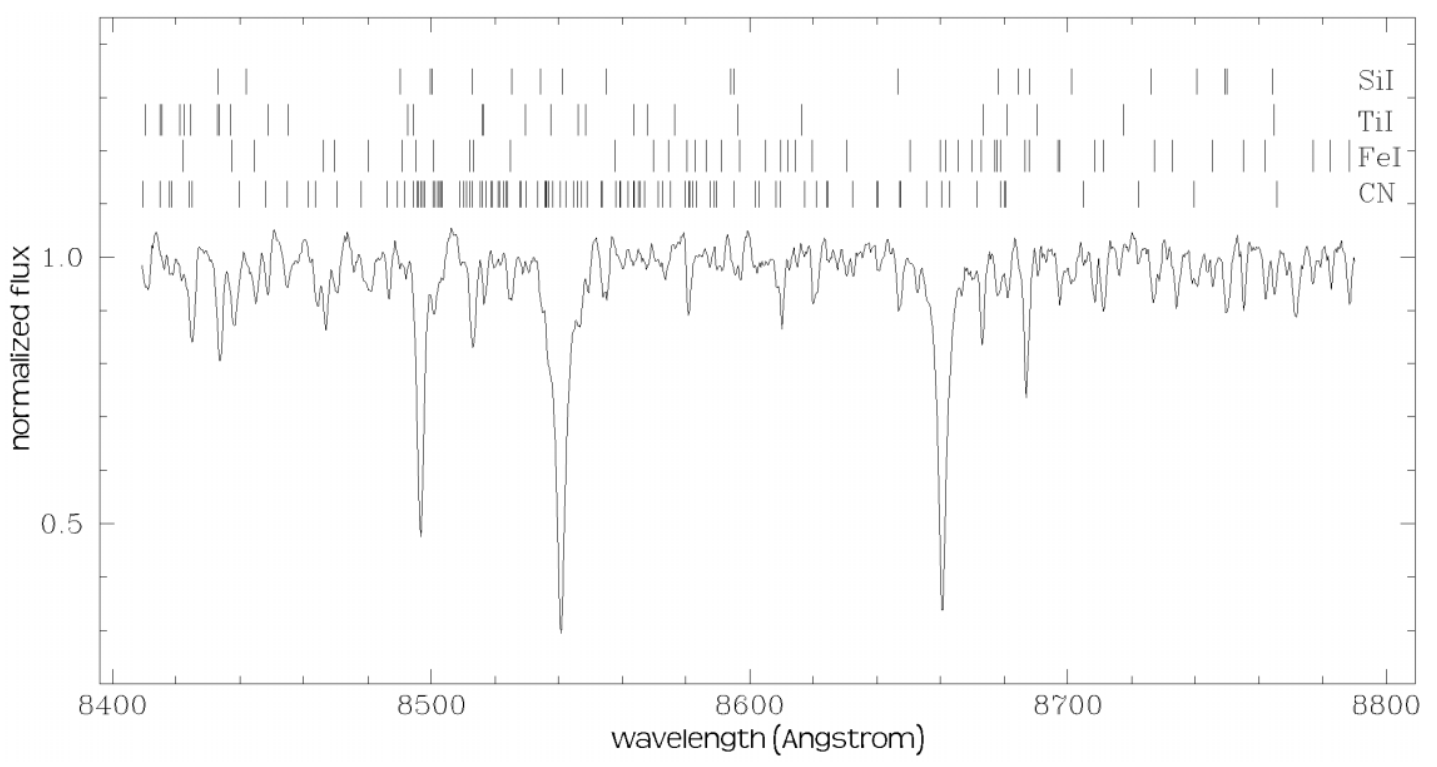

FIG. 1.-RAVE spectrum of a typical field star, HD 154837 (K0 III), illustrating the properties of the chosen wavelength interval around the Ca II IR triplet. The strongest other absorption lines are identified.

7. Calibration of stellar atmospheric parameters and correspondence with the MK scheme through the H-R diagram.

8. Searches for spectroscopic binaries and cataclysmic variables.

9. The $8620 \AA$ diffuse interstellar band as an estimator of interstellar reddening.

In this paper we describe the first data release (DR1) of the RAVE survey, which contains RVs obtained from RAVE spectra (the spectra, stellar parameters, and additional information will be part of further releases, as the first-year spectra are contaminated by second-order light). Photometric and proper-motion data from other surveys are also provided for ease of use. The structure of the paper is as follows: In $\S 2$ we describe the survey layout, technical equipment, and input catalog. Section 3 is devoted to the actual observations, followed by a section detailing the data reduction. Section 5 discusses the data quality and compares RAVE data with independent data taken with other telescopes. Finally, $\S 6$ provides a detailed description of the data product of the DR1 and concludes with longer term perspectives.

\section{SURVEY DESIGN AND INPUT CATALOG}

The wide field of view of the UKST and the multiplexing capability of the $6 \mathrm{dF}$ are well matched to a survey of apparently bright stars. The scientific goals of RAVE include analyzing the chemical and dynamical evolution of the Galaxy, using as tracers both dwarfs and giants observed locally. Most apparently bright stars will be in the thin and thick disks; adopting a limiting magnitude of $I \sim 12$ (see below), dwarfs probe distances of hundreds of parsecs and giants probe out to a few kiloparsecs. With a sufficiently large sample, even apparently bright stars will contain a statistically relevant sample of halo stars.

The most efficient use of the $6 \mathrm{dF}$ is when exposure times on one field match the set-up time for the next field (see below), and this implies a limiting magnitude of around $I=12$. As noted above, RAVE is a precursor to Gaia, and the wavelength range for the RAVE spectra was chosen to match that of the Gaia Radial Velocity Spectrometer (Munari 2003; Katz et al. 2004; Wilkinson et al. 2005), namely, around the Ca II IR triplet. This wavelength range also includes lines due to iron, calcium, silicon, magnesium, and titanium, and detailed analyses should provide an estimate of $[\alpha / \mathrm{Fe}]$, in addition to overall metallicity. Grids of synthetic spectra at a resolution and wavelength range similar to those of RAVE and covering wide ranges of $T_{\text {eff }},[\mathrm{M} / \mathrm{H}]$, $\log g$, and $V_{\text {rot }}$ were calculated by Zwitter et al. (2004) and are shown in their Figure 4.

A typical RAVE spectrum is illustrated in Figure 1. This shows a spectrum of HD 154837 (K0 III) as observed on 2004 September 24, with the continuum normalized to 1.0. The entire wavelength range is dominated by absorption lines; the strongest due to $\mathrm{Fe}$ I, Si I, Ti I, and ${ }^{12} \mathrm{C}^{14} \mathrm{~N}$ are identified. The hump in the continuum around $8508 \AA$ is produced by an opacity minimum, due in particular to the absence of ${ }^{12} \mathrm{C}^{14} \mathrm{~N}$ lines.

This wavelength window implies that an $I$-band selection is most appropriate, and this is indeed the approach taken. Star counts from the DENIS catalog to a limiting magnitude of $I=12$ at typical latitudes and longitudes of RAVE are shown in Figure 2 and show that a few setups with $6 \mathrm{dF}$ per line of sight, with a

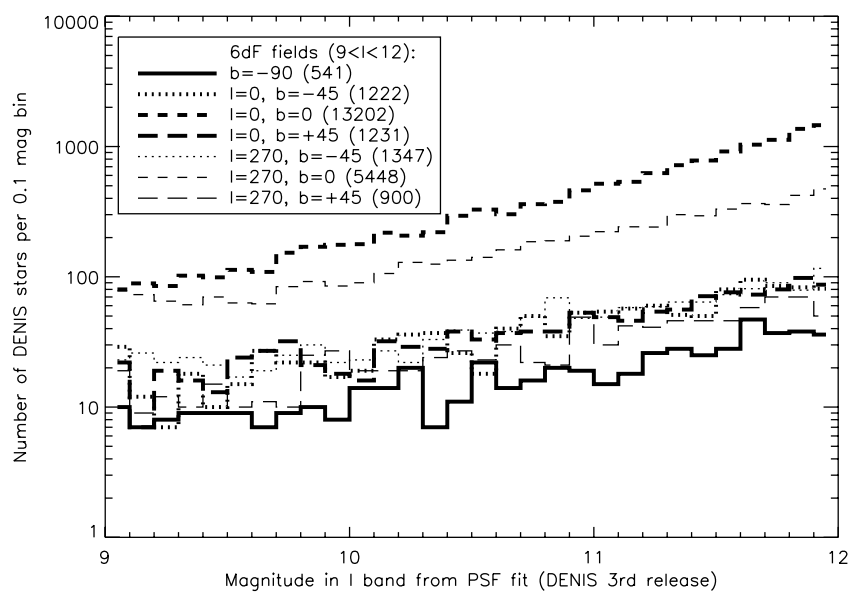

FIG. 2.-DENIS star-count distributions as a function of DENIS I magnitude using the RAVE selection criteria of $9<I<12$ within $6 \mathrm{dF}$ fields of view (5.7 diameter), pointing in the Galactic cardinal directions visible from the Southern Hemisphere. The total number of stars matching the RAVE criteria are given in parentheses in the key to the line types. 
random selection over color, provide a statistically significant sample of the stars on the sky.

\subsection{Description of the Instrument}

The RAVE survey instrument is known as $6 \mathrm{dF}$ (Watson et al. 2000 ) in reference to its $6^{\circ}$ diameter field of view. The $6 \mathrm{dF}$ consists of an off-telescope robotic fiber positioner, two fiber-field plates of 150 fibers each, and a bench-mounted spectrograph that is fed from UKST when one of the field plates is mounted on the telescope. The light received from the target is deflected down the fiber by a $90^{\circ}$ prism contained in a "button" at the end of the fiber. This button is magnetic, allowing secure placement of the fiber on the field plate. The robot uses a pneumatically actuated gripper to pick up and put down the fiber buttons. Each fiber has a diameter of $100 \mu \mathrm{m}(6.7$ on the sky) and can be placed accurately (to within $10 \mu \mathrm{m}$, or 0.7 ) on star positions anywhere within the $6^{\circ}$ diameter field. Each field plate also contains four fiducial fiber bundles of seven fibers arranged in a hexagonal pattern, which are used for field acquisition.

The bench-mounted Schmidt-type spectrograph sits on an optical bench on the floor of the telescope dome. The spectrograph therefore does not suffer from the flexure that affects telescopemounted spectrographs, which are subject to the changing gravity vector as the telescope moves across the sky. Nevertheless, it is sensitive to temperature changes ( $\operatorname{see} \S 5.3$ ). With RAVE we use a specially purchased volume phase holographic (VPH) transmission grating of medium dispersing power; this 1700 lines $\mathrm{mm}^{-1}$ grating is tuned for high efficiency in the $I$ band and identified as grating $1700 \mathrm{I}$. In practice, this setup provides an average resolving power of $\sim 7500$ over the Ca-triplet region, covering the wavelength range 8410-8795 $\AA$.

The CCD used with the $6 \mathrm{dF}$ spectrograph is a Marconi (EEV) CCD47-10-BI detector. It has $13 \mu$ m pixels with a $1 \mathrm{k} \times 1 \mathrm{k}$ format and is thinned and back-illuminated. The actual CCD dimensions are $1056 \times 1027$ pixels. The chip has good cosmetics, with few defects, and has a quantum efficiency of $40 \%-30 \%$ over the wavelength region of RAVE operations (8400-8800 $)$.

The RAVE spectrograph configuration, with the mediumresolution VPH transmission grating, unfortunately exacerbates the effects of the residual aberrations within the Schmidt system. This leads to variable, position-dependent point-spread functions (PSFs) and pincushion distortions. Consequently, the existing pipeline reduction software, which was designed for the lower resolution $6 \mathrm{dF}$ galaxy redshift survey, does not work optimally with RAVE data (but is more than adequate for quick-look, quality control purposes). A dedicated IRAF ${ }^{25}$ pipeline was therefore developed for the reduction of RAVE data (see $\S 4$ for details). Also most VPH gratings exhibit a "ghost," due to light reflected off the detector. This ghost manifests itself as a spurious emission peak in the spectra and cannot be avoided in our RAVE observations. The wavelength of this ghost feature can be pushed into the blue part of the spectra using hardware tuning, and this wavelength region may then be excluded in the data analysis, e.g., when computing the RV using cross-correlation techniques. Not removing this feature properly would result in the correlation function having a strongly asymmetric profile. Fortunately, this effect is only significant in a small fraction of our spectra, and this is noted by a quality flag in the corresponding entries in the data release catalog (see Tables 5 and 7).

\footnotetext{
${ }^{25}$ IRAF is distributed by the National Optical Astronomy Observatory, which is operated by the Association of Universities for Research in Astronomy, Inc., under cooperative agreement with the National Science Foundation.
}

\subsection{Instrument Performance}

Each field plate nominally has 150 target fibers, which when undeployed form a ring around the periphery of the $6^{\circ}$ field. The fibers are evenly spaced around this ring, with the exception of two small gaps at the northern and southern field plate edges. Each gap creates a small "zone of avoidance" that has some (small) impact on the target distributions that can be achieved. Similarly, there is a zone of avoidance, of less significance, associated with the pivot positions of each of the four guide fiber bundles, which are located approximately at the cardinal east-west/north-south points on each field plate. Each target fiber can nominally reach the field center, $+10 \%$, and its deployed location relative to parked is subject to the constraint that the angle from the pure radial direction must be less than $\pm 14^{\circ}$. The targets in a given input field are allocated to a given fiber using a sophisticated field configuration algorithm (FCA) based on that developed for the $2 \mathrm{dF}$ spectrograph (Lewis et al. 2002). The FCA accepts user-supplied priorities within the input target list but does contain subtle allocation biases, as described by P. Outram ${ }^{26}$ and Miszalski (2006).

These subtle allocation biases in the FCA are illustrated for the RAVE targets in Figure 3, which shows contour plots of the successfully allocated targets for the first-year data for each of the two field plates. The contour levels indicate the number of allocated stars per square degree. Since stars are to first order uniformly distributed across the field of view, the nonuniform distribution of allocated targets highlights the inherent bias in the fiber placement. The empty notches at the top and bottom of each field plate are where no fiber buttons are positioned. The central underallocated region is due to the known biases in the CONFIGURE program, by which the central region is considered easy to reach and therefore left until after harder targets, close to the field edge, have been allocated. Often this leaves fewer fibers than targets available for the center. The remaining structure in these figures is the result of fibers being unavailable (e.g., due to breakages) or having a shorter fiber length available (after repair), reinforcing the central deficiency.

Over the current survey lifetime, both field plates have had, on average, 130 fibers available for allocation, an extremely consistent overall level for each. The $\sim 15 \%$ of fibers that are unavailable for allocation at any one time are due to a variety of causes. The most common problem is fiber breakages, which are largely the result of retractor problems on parking the fiber. Another problem is fractures in the fibers themselves (due to buttons "stamping" on them during fiber placement or having the trailing short metal shank of the fiber bent on parking via collisions with the pivot point). Fractures may still allow light to be transmitted but lead to severe fringing effects in any resulting spectrum. Another problem is throughput deterioration of the fibers, possibly due to a gradual clouding over time of the UV curing optical adhesive used to mate fibers to prisms (as was the case for the earlier UKST MOS system FLAIR [Parker \& Watson 1990]). The final problem is related to the fiber-button gripper mechanism, which can lead to the robot being unable to pick up or put down some fibers.

Among the problems listed above, the first two are linked to the integrity of the fibers and can be solved by repairing them. Fiber repairs are a difficult and time-consuming process that usually involves a shortening of the fiber itself, which after several repairs can reduce its effective range. Nevertheless, considerable effort has been expended to maintain the multiplex gain of each field plate.

\footnotetext{
${ }^{26}$ See http://www.aao.gov.au/local/www/brent/configure.
} 

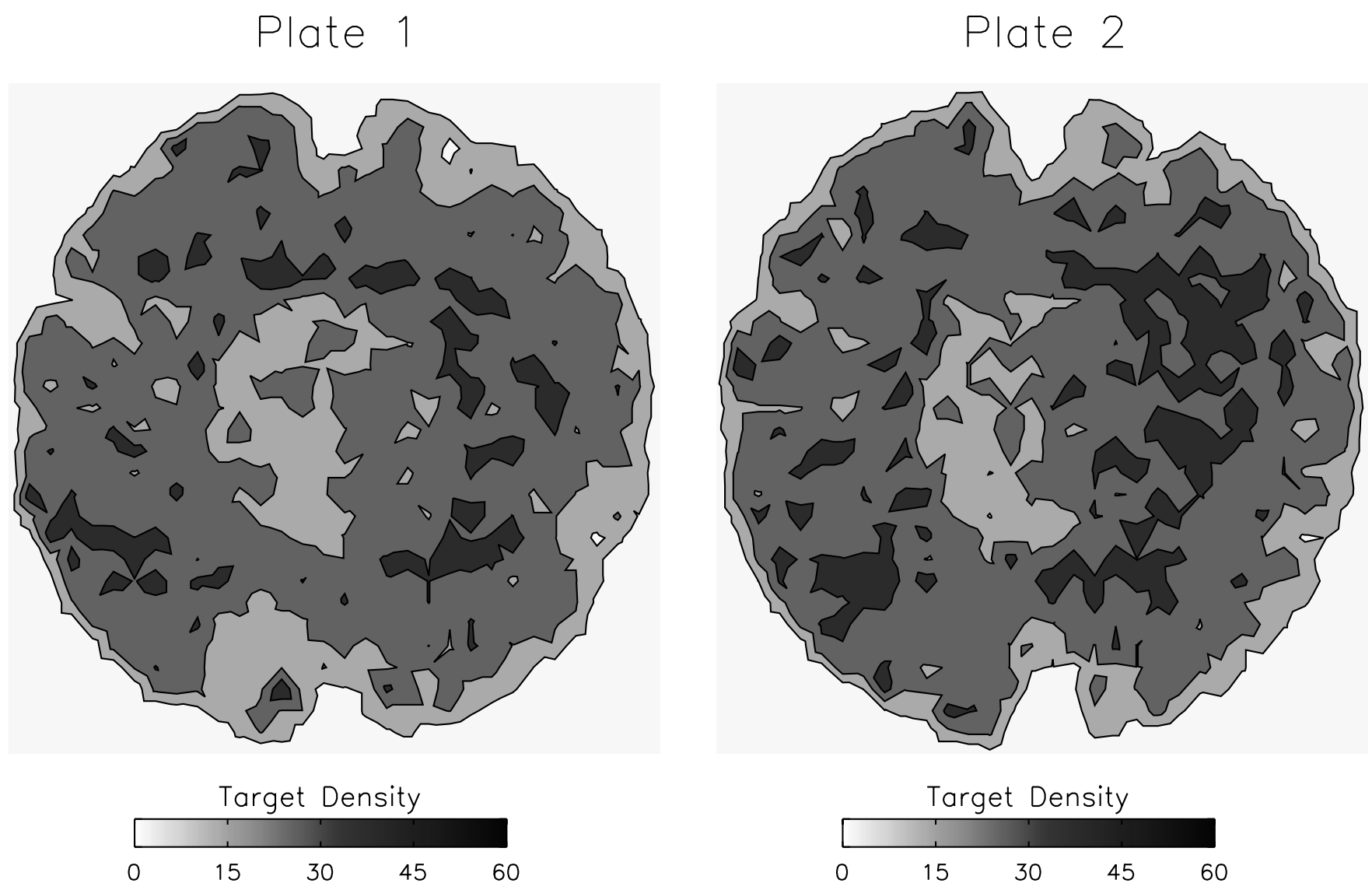

FIG. 3.-Density of successfully allocated targets across each field plate from RAVE's first-year data. The contours are at 15,30 , and 45 allocated targets per square degree. The structure visible in each plot is due to (1) the northern and southern fiber gap across each plate, (2) zones of avoidance around each of the four equally spaced guide fibers, (3) known biases within the CONFIGURE program (most obvious of which is the central left-hand underdensity), and (4) broken fibers. [See the electronic edition of the Journal for a color version of this figure.]

\subsection{Sample Selection and Input Catalog}

The original RAVE sample was intended to be magnitudelimited over the range $9<I<12$, with no color selection (where $I$ refers to Cousins $I$ ). At the time of the initial stages, data from the Two Micron All Sky Survey (2MASS) and the DENIS survey were not yet available, and the RAVE target stars were drawn from the Tycho-2 catalog of the 2.5 million brightest stars (Høg et al. 2000) and the SSS (Hambly et al. 2001b). The Tycho observations were made in two nonstandard filters, $B_{\mathrm{T}}$ and $V_{\mathrm{T}}$. These passbands were converted to Johnson $B$ and $V$ using the transformation (Perryman et al. 1997)

$$
\begin{gathered}
V=V_{\mathrm{T}}-0.090\left(B_{\mathrm{T}}-V_{\mathrm{T}}\right), \\
B=V+0.850\left(B_{\mathrm{T}}-V_{\mathrm{T}}\right) .
\end{gathered}
$$

Here $(B-V)$ was transformed to $I$ using an empirically derived zeroth-order mean color transformation derived from Bessell (1979) Tables 2 and 3:

$$
\begin{gathered}
(V-I)=1.007(B-V)+0.03 \quad \text { if } \quad(B-V)<1.30 \\
(V-I)=2.444(B-V)-1.84 \quad \text { otherwise. }
\end{gathered}
$$

The SSS photometry (Hambly et al. 2001a) is photographic $I$ (IVN emulsion, hereafter $I_{\mathrm{IVN}}$ ), extracted from the SRC-I UKST IVN Survey plates by SuperCOSMOS, the automatic plate-scanning facility at the Royal Observatory, Edinburgh. Blair \& Gilmore (1982) showed $I_{\mathrm{IVN}}$ to be directly equivalent to
Cousins $I$, essentially without any color correction, with a relation between the two magnitudes of

$$
I-I_{\mathrm{IVN}}=0.00 \pm 0.03(V-I) .
$$

The $11<I<12$ interval of the first-year input catalog consists only of SSS stars. Stars with $9<I<11$ are mainly from Tycho-2 but also include SSS stars that do not appear within $7^{\prime \prime}$ of any Tycho- 2 star (this requirement is set by the $6 \mathrm{dF}$ fiber diameter of 6."7). Any SSS star that does appear within 7" of a Tycho-2 star is not included, and neither is the Tycho-2 star. This procedure allows us to avoid any contamination by a possible nearby star. The quality of the photometry (not the astrometry) in both catalogs is not sufficient to discriminate between the two possibilities, either that (1) the two are in fact the same star measured to have different positions in each catalog, or (2) the two are in fact two different stars. The criteria for inclusion in the input catalog are designed to merge the two samples, taking into account both their incompletenesses at $I \sim 11$ (the faint end of Tycho-2 and the bright end of SSS) and the better proper-motion accuracy provided by Tycho- 2 . As a result, the interim input catalog contained $\sim 300,000$ stars with $\sim 50 \%$ Tycho- 2 and $\sim 50 \%$ SSS.

We selected 478 contiguous survey fields to cover $\sim 12,200 \mathrm{deg}^{2}$ of the southern sky visible from the UKST, excluding regions with Galactic latitude $|b|<14^{\circ}$ to minimize dust obscuration and crowding. The field centers are defined on a $5^{\circ} .7$ grid spacing, corresponding to the field of view of the $6 \mathrm{dF}$ field 
plates. The gaps between the fixed circular tiling scheme miss about $20 \%$ of the available area. For each field of view, 400 targets were randomly selected from the input catalog to construct two field files, consisting of 200 stars in each, so that at least two separate $6 \mathrm{dF}$ pointings could be made.

No subselection into bright and faint samples was made for the first $2.5 \mathrm{yr}$ of the RAVE survey, including the observations for this first data release. Occasionally this leads to, for example, a 9 mag star being adjacent on the slit to a significantly fainter 12 mag star. The tight spacing of the 150 fibers along the slit, as imaged onto the CCD, means that about $4 \%$ of each spectrum's flux contaminates that of the adjacent fibers (this effect is known as fiber cross talk). This is an insignificant problem for RV determinations for the vast majority of our targets but can impact the abundance determinations, especially when the apparent magnitude difference between two adjacent fibers is large. This crosstalk effect is carefully taken into account by the reduction pipeline using iterative cleaning (see $\S 4.2$ ).

Typically, there are $\sim 200$ potential targets for each field pointing at the RAVE magnitude limits, which ensures efficient fiber configurations. The $6 \mathrm{dF}$ CONFIGURE software is usually able to allocate all the available science fibers to targets, unless two target stars are closer together than the minimum allowed separation dictated by the size of the $6 \mathrm{dF}$ button footprint on the field plate (approximately $5^{\prime}$ ). For the first year of RAVE, typically $\sim 130$ fibers are allocated to target stars for a single pointing. This offers scope for two pointings on some fields and reobservation of selected targets (repeats).

Both Tycho-2 and SSS are primarily astrometric catalogs and so only provide approximate photometry. The second DENIS data release, made public in $2003,{ }^{27}$ presented the first opportunity to compare the input catalog $I$ directly with more accurate $I$-band photometry (better than $0.1 \mathrm{mag}$ ). As noted earlier, $I_{\mathrm{IVN}}$ is equivalent to standard $I$. Bessell (1986) showed that there is essentially no difference between Gunn- $i$ (DENIS $I$ ) and $I_{\text {IVN }}$, allowing a direct comparison of input catalog $I$ with DENIS $I$. Figure 4 (top) shows that the Tycho-2 I magnitudes derived from the Tycho- $2 B_{\mathrm{T}}$ and $V_{\mathrm{T}}$ photometry systematically agree with DENIS $I$ (mean offset $=-0.095 \mathrm{mag}$ ), albeit with a large scatter $(\sigma=0.385 \mathrm{mag})$. The SSS I magnitudes appear to be nonlinear, systematically diverging from the DENIS $I$ with brightness (mean offset $=-0.50 \mathrm{mag}, \sigma=0.33 \mathrm{mag}$ ). DENIS provides the first large-scale, external check on the accuracy of the SSS photometry for bright stars. This diverging offset reflects the SSS zero-point error in linearizing the nonlinear saturation of bright stars on photographic plates. A result is that the number density of SSS stars in our sample, shown in Figure 4 (top), actually peaks fainter than the planned selection window.

Figure 4 (bottom) compares a subsample of observed RAVE stars with the number of DENIS stars in the same area of sky as a function of DENIS I magnitude. The solid line emphasizes that RAVE is not complete. The broken lines highlight color biases present in the input catalog. The bright sample $(I<11$, mainly Tycho-2) has relatively more blue stars and fewer red stars than the faint sample ( $I>11$, entirely SSS), and vice versa, reflecting the effective selection biases in the two subsamples. Although the pseudo-I-band selection from Tycho-2 has reduced Tycho-2's $B$-selection bias, the bias is still visible in Figure 4, compared to real $I$-band selection from SSS. Hence, the input catalog is not a homogeneous selection window: sample-dependent color biases

\footnotetext{
27 The latest DENIS release in 2005 is available at the CDS using the VizieR facility. At the time of this study, only the second incremental release was available.
}
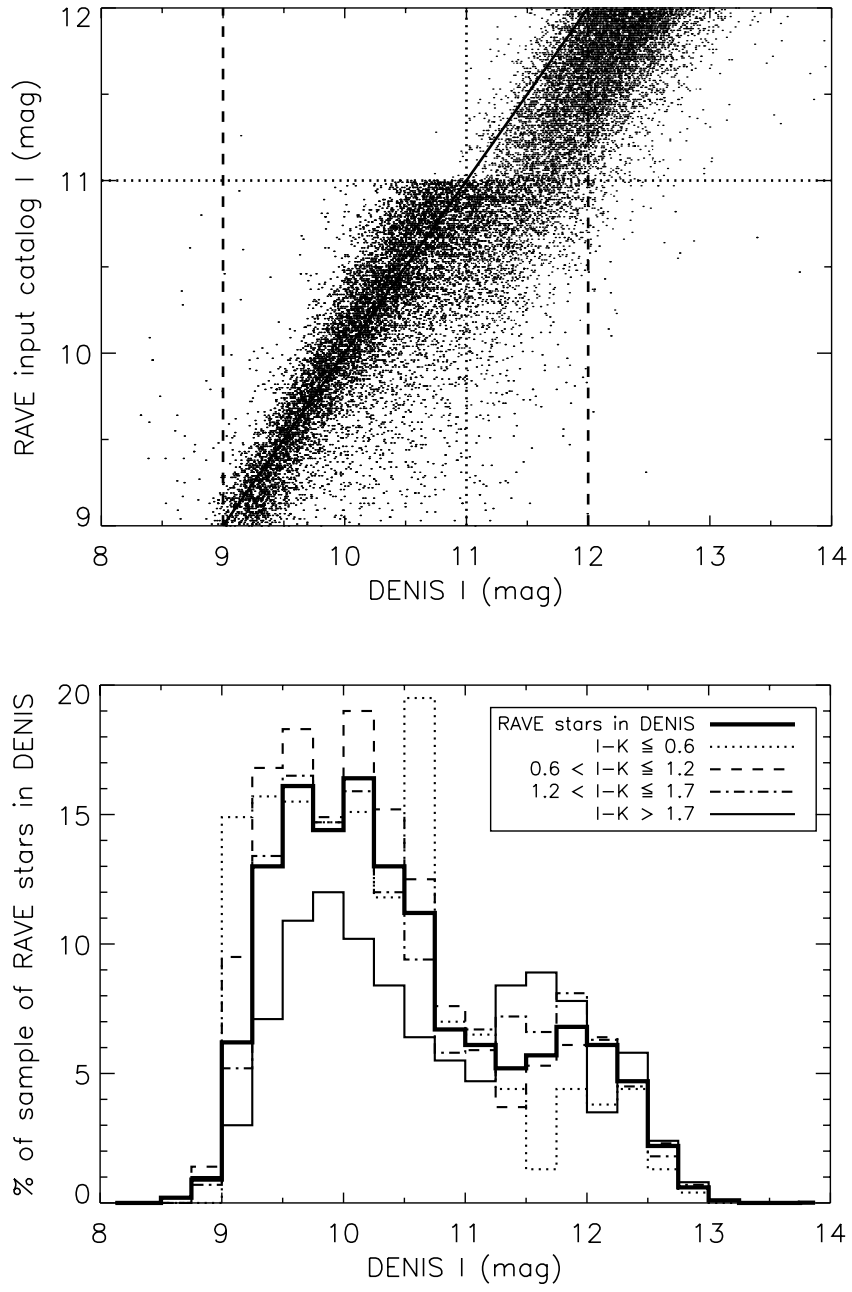

FIG. 4.-Top: Input catalog I magnitudes (Tycho-2 stars entirely within vertical axis $I<11$ and SSS predominantly within vertical axis $I>11$ ) plotted as a function of DENIS $I$ for a subsample of observed RAVE stars that are also in the second DENIS data release. The dashed lines represent the planned selection window on each axis. The dotted line delimits the bright and the faint samples at $I=11$. Bottom: Histogram of RAVE completeness compared to DENIS, as a function of DENIS $I$ magnitude and DENIS $I-K$ color.

exist. In terms of stellar population studies, one of RAVE's scientific goals is to target red giants, $1.2<I-K \leq 1.7$, to probe the Galactic disk and halo. This goal has not been compromised by the photometry on which the interim input catalog was based.

To summarize, the catalog has no kinematic bias, but Galactic science using RAVE requires care to account for the various selection biases introduced by the inhomogeneous photometry used to derive the input sample. We must stress that the RAVE catalog in the first data release is clearly incomplete within its selection criteria (and was not intended to be complete) and has subtle color biases. The distribution of RAVE stars in the color-magnitude diagram is not entirely representative of the underlying Galactic population due to the selection effects described above. Subsequent data releases will include stars selected by $I$ magnitude from the DENIS catalog, which should provide an essentially unbiased representative sample of Galactic stars in the selected range of magnitude.

Figure 5 compares the $I$-band apparent magnitude distributions of the RAVE sample and the Hipparcos-based GCS sample (Nordström et al. 2004), which is the largest previously published sample of accurate stellar RVs. The RAVE I magnitudes for the brighter RAVE stars and the GCS sample are derived from $B$ and 


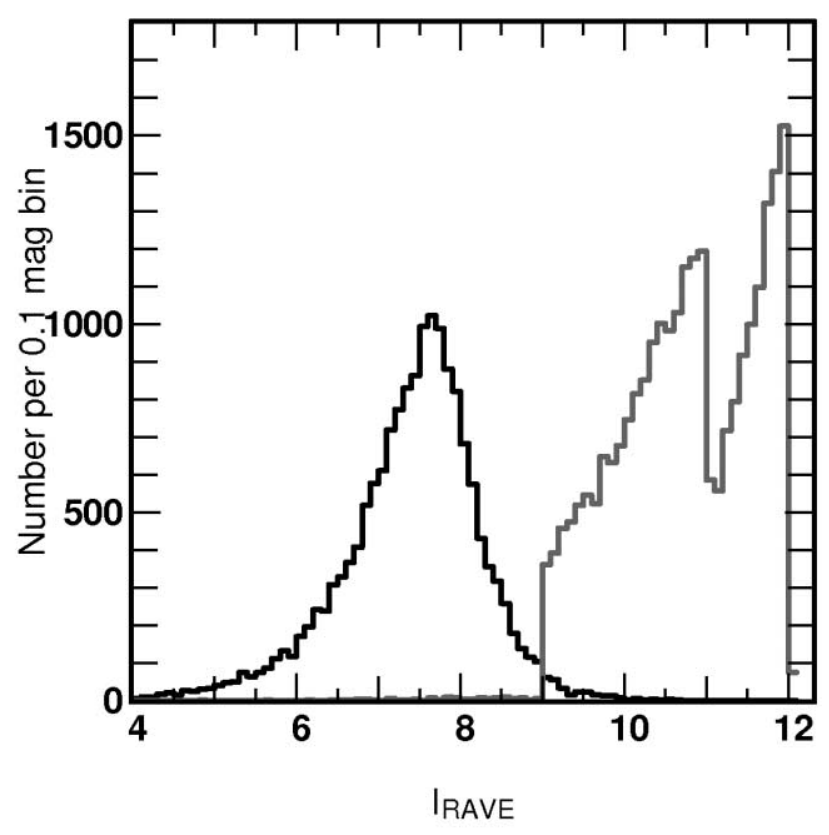

FIG. 5.-Comparison of RAVE (gray) and GCS (black) apparent magnitude distribution, showing that RAVE is significantly fainter than the previous large RV survey. The GCS data (Nordström et al. 2004) have been transformed to RAVE's input catalog $I$-band magnitude using eqs. (1) and (2).

$V$ magnitudes using equations (1) and (2). The RAVE and GCS samples hardly overlap in apparent brightness. The RAVE magnitude range $(9<I<12)$ was chosen to match the scientific goals of the RAVE survey with the instrumental capabilities of the $6 \mathrm{dF}$ system. Therefore, as shown in Figure 5, the RAVE sample is much fainter than the GCS sample. This will be relevant when we compare the RAVE velocities with those from external data sets $(\S 5.6)$.

\subsubsection{RAVE Catalog Contamination}

Although RAVE target samples are ostensibly single stars selected from Tycho and SSS I, both samples, including Tycho, do in fact contain double-star contaminants. This was noticed during the early part of the survey from a casual examination of printed target images. There are three main situations that arise: (1) Close doubles of similar magnitude for which the $6 \mathrm{dF}$ fiber will be positioned on the center of the double image and a composite spectrum will result. (2) Close pairs in which one star is very much brighter than the other. The position for these "binaries" will be heavily weighted to the bright component, and so only a small effect will result. (3) Relatively close pairs (and this is a situation that only arises with the SSS data at the fainter end) for which a blended SSS image results but the SSS position is between the two stars in the blend. In such cases the fiber would be positioned essentially on sky. An additional case of multiple blends of three or more stars is so rare that it can be ignored. To assess the extent of the problem, 200 randomly selected fields from the first-year data were chosen, and each "thumbnail" small-area image for each target was carefully examined. This established that the problem was present at a maximum of $0.4 \%$ level. As a further check, a blind comparison of these contaminants was made with the associated spectra to see whether such blends were obvious. Only a fraction $(25 \%)$ of the problem stars were obvious in the spectra. Now we have a rigorous regime in which all candidate stars are checked for possible contamination prior to observing, using the $1^{\prime}$ SSS thumbnails from the online SSS $R$-band data. Such contaminants are identified and removed from the database.

\section{OBSERVATIONS}

Observing time during the first year of the RAVE survey was confined to an average of seven bright/gray nights per lunation, distributed around (but not including) four or five unscheduled bright-of-moon nights. The project began on 2003 April 11, and the first year's data include spectra obtained between then and 2004 April 3, for a total of 88 scheduled nights.

Observations for RAVE consist of a sequence of target field exposure, arc, and flat. During the first year, $\mathrm{Ne}, \mathrm{Rb}$, and $\mathrm{Hg}-\mathrm{Cd}$ calibration exposures were obtained for each field, together with a quartz flat field for spectrum extraction in the data reduction. All calibration exposures were obtained by reflection from a white full-aperture diffusing screen located close to the entrance pupil of the telescope (the Schmidt corrector). In this position, the effects of irregularities in the screen illumination are minimized, while the fibers are illuminated at a focal ratio $(f / 2.5)$ similar to that of the incoming starlight. The requirement for arc and flat observations to be obtained for each field arises because the slit units are interchanged in the spectrograph for each new configuration (along with the field plates in the telescope).

Several target fibers are reserved to monitor the sky for background subtraction. As RAVE stars are quite bright, only a small fraction of the signal in each fiber comes from the sky. In most cases, only sky emission lines are present, with little or no background of scattered solar spectrum from the Moon, despite the fact that most first-year observations were obtained in bright or gray time. It is thus necessary to subtract this sky using measurements from dedicated sky fibers, placed uniformly across the field. A calculation of the optimal number of sky fibers per plate is given in Kuijken \& Gilmore (1989b), while a detailed description of sky subtraction with fibers is given in Wyse \& Gilmore (1992). Each of the RAVE target frames therefore contained spectra of at least 10 sky samples, obtained using dedicated sky fibers. These were combined and scaled in the reduction process for sky subtraction.

On a subset of nights (of order 1/4), twilight exposures were also taken to provide additional zero-point velocity checks from solar absorption features and twilight sodium $\mathrm{D}$ lines. These were obtained with the telescope pointing toward the zenith and were taken within 30 minutes of sunset or sunrise. Those twilights frames are particularly useful to assess the zero-point stability and enable some more RV checks.

The target frames themselves consist of five consecutive exposures of $600 \mathrm{~s}$ each, allowing adequate signal-to-noise ratio $(\mathrm{S} / \mathrm{N})$ to be obtained in the summed spectra, while minimizing the risk of saturation from particularly bright stars. In poor conditions of low sky transparency, additional exposures are made. The total time for a "standard" set of arc, flat, and field exposures is of order $1 \mathrm{hr}$, which is similar to the fiber reconfiguration time in the $6 \mathrm{dF}$ robot. Thus, while observing proceeds with one field plate, the other is being reconfigured, maximizing the time-ontarget productivity of the telescope. Taking account of the physical transportation and exchange of the field plates, the slew time for the telescope, field acquisition, etc., an experienced observer is able to accumulate acceptable data for up to eight RAVE fields on a midwinter's night at the latitude of Siding Spring Observatory.

The median seeing at the focal surface of the UKST (i.e., including local site effects and convolved with the instrumental PSF) is $\sim 2$ ".5. This is much less than the 6.7 diameter of the fibers, and so some seeing degradation is possible without serious loss of flux. In extremely poor conditions, however (seeing $\sim 4^{\prime \prime}$ ), 
there is a noticeable loss of signal. This is principally due to the difficulty in obtaining perfect target acquisition because the seeing degrades spatial resolution in the guide fiber bundles. Fortunately, such poor seeing is relatively rare $(\sim 10 \%-15 \%$ of clear time).

During the first year of RAVE operations, no blocking filter was used to isolate the spectral range required, so there is secondorder contamination of the spectra. Flux in the approximate wavelength range $4200-4400 \AA$ is therefore added to that in the primary spectral range (8410-8790 $\AA$ ). In practice, this is only a serious problem for hotter stars (earlier spectral types than $\sim \mathrm{F}$ ), but features such as $\mathrm{H} \gamma$ will be present at a low level in all stars.

The final tally for the first year's observations was 24,748 target stars in 240 fields. Of these stars, 24,320 were observed once, 330 were observed twice, and 98 were observed three times. A small subset of these stars $(\sim 100)$ were also observed with the $2.3 \mathrm{~m}$ telescope of the Australian National University at Siding Spring Observatory and the ELODIE echelle spectrograph at the Observatoire de Haute-Provence (France), and a further small number was found to be part of the GCS sample. Those observations are described in more detail in $\S 5.6$.

\section{DATA REDUCTION}

Data reduction for the RAVE project consists of two phases. First, a quick-look data reduction is carried out at the telescope using AAO software. This was originally the $2 \mathrm{dFdr}$ package developed for the $2 \mathrm{dF}$ Galaxy Redshift Survey. It was modified to reduce $6 \mathrm{dF}$ data for the $6 \mathrm{dF}$ Galaxy Survey (therefore named $6 \mathrm{dFdr}$ for $6 \mathrm{dF}$ data reduction software). At RAVE's high dispersion, the wavelength modeling of $6 \mathrm{dFdr}$ was too crude to allow arc lines to be correctly identified. Therefore, the spectrograph optics model in $6 \mathrm{dFdr}$ has been upgraded to allow correct line identification at all dispersions.

The $6 \mathrm{dFdr}$ software was used to reduce RAVE data using simple fiber extractions from the raw data frames (summing pixel values over a fixed width around each fiber trace) without subtracting scattered light. The $6 \mathrm{dFdr}$ software was upgraded to RAVEdr in 2004 November to include scattered light subtraction. Note that RAVEdr (and 6dFdr) is able to perform more sophisticated fiber extractions, which fit profiles to each fiber cross section to reduce fiber cross talk. However, this critically depends on the accurate subtraction of scattered light. The current scattered-light model is only valid for all stars of equal brightness, which is not always the case for RAVE's observations. In addition, RAVE's bright star observations cause the more sophisticated fiber extraction to be unstable, so the simple method is used. This results in fiber cross talk being not optimally modeled with RAVEdr. Therefore, an IRAF-based data reduction pipeline has been built to reduce RAVE observations. This pipeline is described in $\S 4.1$. Nevertheless, the RAVEdr reductions by the UKST observers remain useful as the first of many data-quality checks.

\subsection{Raw Data Reduction}

Raw data are reduced with a custom IRAF pipeline. The pipeline uses three types of exposures to reduce every field: a set of scientific exposures is followed by a neon arc and a flat-field exposure. The flat-field frame is used to identify the location on the CCD of the different spectra of individual fibers. This is done automatically, using the information that there are small additional gaps following the fibers with numbers 50 and 100. The pixels associated with all fibers exceeding a threshold signal are extracted. This allows the evaluation of fiber cross talk and scattered light for any fiber with contributing light, even if it has been identified as broken.
The fibers are relatively close to each other on the CCD, with a typical fiber-to-fiber separation being approximately twice the FWHM of their spatial PSF profile. Fiber cross talk therefore needs to be analyzed and included in the reduction procedures. We adopted the following procedure: first, all light within \pm 0.7 FWHM of a given fiber center is attributed to this fiber. Next, a Gaussian modeling the contribution from the neighboring fibers is subtracted, and then the whole procedure is iterated twice. We estimate, from our experience, that in the final spectrum less than $1 \%$ of the flux is due to stars in adjacent fibers along the slit, even if those adjacent stars are 2.5 mag brighter. This level of residual contamination is certainly acceptable.

The scattered light background requires a different careful treatment. It is relatively strong, and there are no large gaps in the distribution offibers on the entrance slit that allow for its easy determination. The scattered light is modeled by spreading a fraction of the total stellar light to a wide, axially symmetric Gaussian profile (see Wyse \& Gilmore 1992 for discussion). Its width is $\sim 1 / 3$ of the CCD chip size and its intensity $\sim 13 \%$ of the stellar flux. These two parameter values are determined iteratively by checking the residuals in flat-field and scientific exposures. Spectra of sky fibers (background) typically feature only sky emission lines ( $\sim 90 \%$ of the cases), so their zero-continuum value is used as a further check on the consistency of the scattered-light model. In the remaining $10 \%$, scattered light from cirrus clouds and/or moonlight add an absorption spectrum to the sky spectra (resulting in a nonzero continuum). Those cases are easily detected via the existence of calcium absorption lines in the spectra. Here we check that the $\mathrm{Ca}$ lines have meaningful intensities (e.g., counts at the center are nonnegative).

Other tasks performed by the IRAF pipeline include flatfielding, wavelength calibration, sky subtraction, and heliocentric correction. Flat-fielding is crucial, as fringing is very strong at the wavelengths of RAVE spectra. Wavelength calibration is achieved by a low-order polynomial fit to nine Ne arc lines. The sky to be subtracted from all spectra is determined as a median of those sky fiber spectra that are found to be free from stellar contamination, fiber cross talk, or ghost peaks.

The observed wavelengths are corrected for the rotation of the Earth, the motion of the Earth about the Earth-Moon barycenter, and the orbit of the barycenter about the Sun. The corresponding formulae are given in the help file of the procedure rvcorrect in IRAF. The resulting transformation from observed to heliocentric velocities should be accurate to $0.005 \mathrm{~km} \mathrm{~s}^{-1}$.

A median combination of a sequence of five $600 \mathrm{~s}$ exposures produces the final spectrum with improved $\mathrm{S} / \mathrm{N}$ and essentially free from cosmic-ray hits (except for cosmic-ray hits in the $250 \mathrm{~s}$ exposure of the flat field). Values of all parameters used during reduction are recorded in the reduced file's header, while any peculiarities are noted in the appropriate reduction logs.

The procedure is automated, but the user is asked to manually confirm the crucial steps: fiber identification, scattered-light subtraction parameters, quality of wavelength solution for each fiber, and the appearance of fibers with sky (background) spectra. This ensures that errors due to problems with the weather or the instrument do not compromise the results. An experienced user needs $\sim 20$ minutes to reduce the sequence offive scientific exposures of a given field, together with their flat-field and arc exposures.

\subsection{Radial Velocity}

\subsubsection{Rest-Frame Radial Velocity Measurement}

The measurement of RVs is performed by an automatic pipeline (see Fig. 6 for a schematic description) that uses a standard 


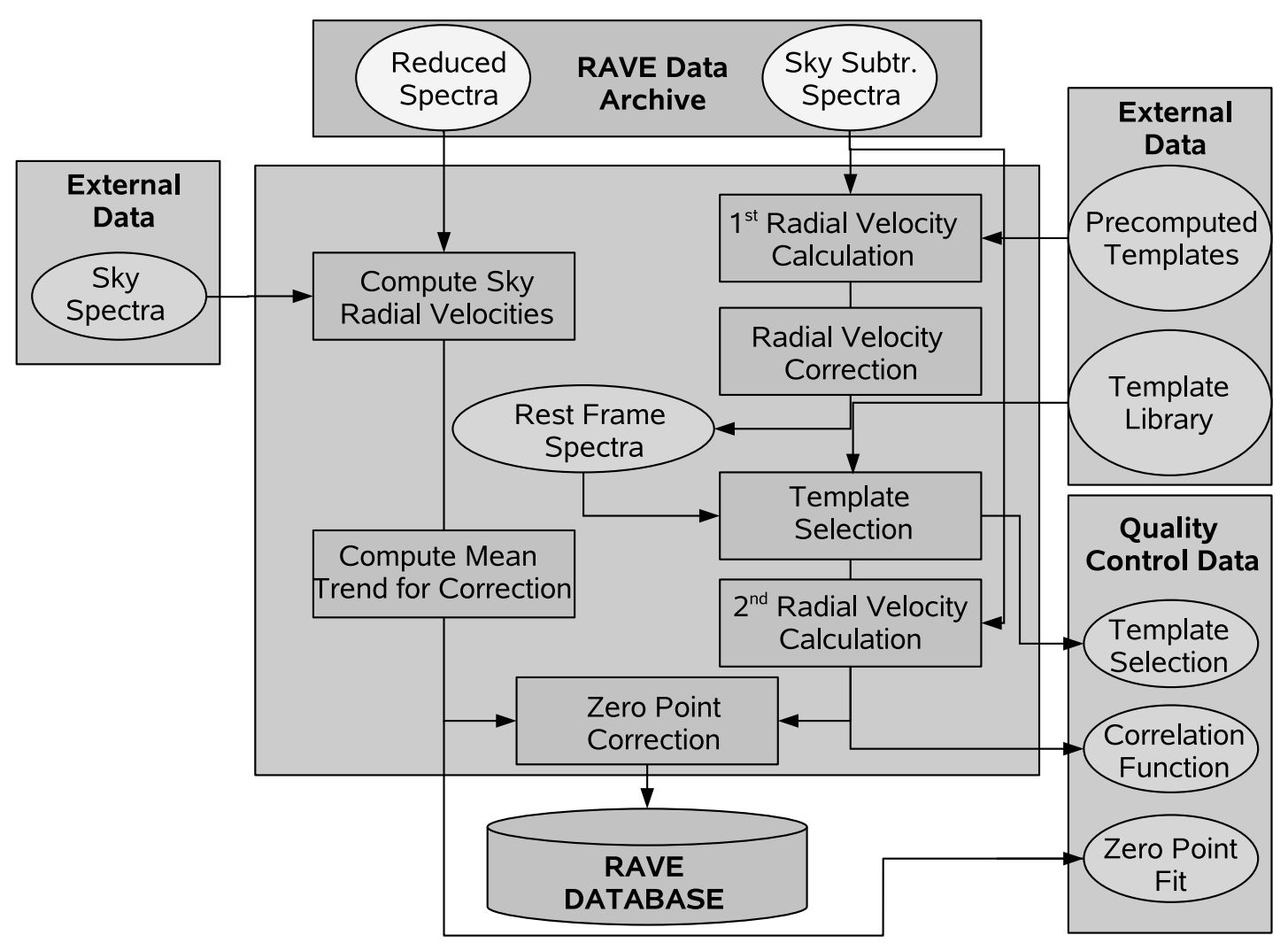

Fig. 6.-Description of the RV pipeline. The pipeline is made of two main branches; the first one (on the right) computes the RVs from sky-subtracted spectra, while the second branch (on the left) uses sky-unsubtracted spectra to compute the zero-point correction. [See the electronic edition of the Journal for a color version of this figure.]

cross-correlation procedure (Tonry \& Davis 1979). The speed of such routines, based on Fourier transforms, makes them well adapted to large data sets like RAVE, in which many RV derivations have to be performed. Here the pipeline uses the package XCSAO for IRAF (Kurtz et al. 1992), as it combines both the speed of the cross-correlation techniques and adequate formats for the outputs.

When computing the RVs, the blue and red ends of the spectra are rejected, the effective wavelength interval used being 8460 $8746 \AA$. The reason for this is to avoid the poorer focus, lower resolving power, and lower quality of the wavelength calibration at the edges of the spectral interval while also excluding, in most of the cases, the emission ghost feature at $\sim 8450 \AA$ that was discussed in $\S 2.1$.

Prior to the RV determination, the spectra are also continuumnormalized using a cubic spline. A cosine-bell filter is used to minimize the effects of (high-frequency) noise, and the RV is calculated by fitting a parabola to the top of the correlation peak (the top $20 \%$ of the correlation function is used throughout the pipeline).

Measuring RVs using cross-correlation techniques relies on the availability of accurate stellar templates to correlate with the observed spectra. Generally, one template or a reduced set of templates is used so as to match a given spectral type. ${ }^{28}$ As the RAVE sample covers the entire color-magnitude diagram and therefore includes large ranges of stellar physical properties such as $T_{\text {eff }}$, $\log g,[\mathrm{M} / \mathrm{H}]$, and rotational velocity, a large number of templates

\footnotetext{
${ }^{28}$ For example, the automatic reduction pipeline for the high-resolution echelle spectrograph ELODIE at the Observatoire de Haute-Provence (used mainly for an extrasolar planet search) uses two templates, matching $\mathrm{G}$ and $\mathrm{K}$ dwarfs.
}

is needed in order to obtain accurate RVs for all targets. We use the library of theoretical spectra from Zwitter et al. (2004) covering the RAVE wavelength interval. This library contains 62,659 synthetic spectra at the RAVE/Gaia resolution, constructed using the Kurucz model atmosphere code (ATLAS). It covers an extensive range of physical conditions of the stellar atmosphere.

As no blue-blocking filter (OG531) was used during the first year of observations, the resulting second-order contamination needs to be taken into account. The second-order templates (requiring a bluer wavelength range than the Zwitter et al. [2004] spectra) are taken from the Munari et al. (2005) library, which contains 28,180 synthetic ATLAS spectra that have been degraded to RAVE's resolution. The combination of both libraries leads to a set of 22,992 synthetic spectra that are used for this first release. The range in effective temperature of this library is $3500 \mathrm{~K} \leq$ $T_{\text {eff }} \leq 40,000 \mathrm{~K}$, giving good coverage of all spectral types. The value of $\log g$ varies from 0 to 5 in steps of 0.5 , while the metallicity $[\mathrm{M} / \mathrm{H}]$ is computed for $[-2.5,-2,-1.5,-1,-0.5,-0.2,0$, $0.2,0.5]$, all scaled solar elemental abundances. The rotation velocities range from 0 to $500 \mathrm{~km} \mathrm{~s}^{-1}$, with irregular spacing. For the entire library of synthetic spectra, the microturbulent velocity is assumed to have a value of $2 \mathrm{~km} \mathrm{~s}^{-1}$.

The number of template spectra is sufficiently large that it is not possible to compute the correlation function for all of them. Therefore, the RVs are obtained using a four-step process. In step 1, we perform a first guess of the RV using a reduced set of 40 templates evenly covering the parameter space of $T_{\text {eff }}, \log g$, and the ratio of first to second order (matching spectral type in the template is more important for RV determination than is metallicity). This first estimate provides a median internal accuracy of $\sim 5 \mathrm{~km} \mathrm{~s}^{-1}$ for most of the Ca II-dominated spectra. For early-type 
stars, for which the Paschen lines are dominant, the accuracy is lower due to the large width of those hydrogen lines. As a comparison, the pixel size in velocity space for a typical RAVE spectrum varies from 12 to $14 \mathrm{~km} \mathrm{~s}^{-1}$ from the red to the blue, with a mean value of $13 \mathrm{~km} \mathrm{~s}^{-1}$. Therefore, with careful analysis and high $\mathrm{S} / \mathrm{N}$, we can expect to reach an $\sim 1.3 \mathrm{~km} \mathrm{~s}^{-1}$ internal accuracy for the RVs (1/10 of a pixel in velocity space).

In step 2, using this first estimate of the RV, the observed spectrum is shifted to the rest frame and compared to the full template database to select the "best-matching" synthetic spectra. The bestmatching template is defined as the spectrum (a combination of one first-order synthetic spectrum and one second-order synthetic spectrum) that minimizes the quantity

$$
D=\sum_{\lambda}\left\{O(\lambda)-\left[(1-c) S_{1}(\lambda)+c S_{2}(\lambda)\right]\right\}^{2}
$$

where $O(\lambda)$ is the continuum-normalized observed target spectrum, $S_{1,2}$ are the continuum-normalized first- and second-order templates, and $c$ is the fractional second-order contamination.

This equation does not account for a color term that is expected because of the shape of the continuum between the first- and second-order wavelength ranges. Nevertheless, this effect is small (due to the limited range in wavelength of the RAVE spectra, only $\sim 350 \AA$ ) compared to other sources of error, such as noise in the observed spectra or residual scattered light. Furthermore, including a color term properly would require continuum normalization for each possible combination of $S_{1}, S_{2}$, and $c$, which is beyond the computational time limit for the pipeline. Finally, to speed up selection of the best template, $c$ is not treated as a continuous variable but rather is limited to discrete values between 0 and 0.8 , spaced by 0.1 , covering the possible values of this parameter. An example of the acceptable fit that is the outcome of this templatefitting procedure is given in Figure 7. The estimated contribution of second-order light to the observed spectrum is indicated, plus the small residuals after the template are subtracted from the observed spectrum.

At step 3, the appropriate template having been chosen, a new $\mathrm{RV}$ is calculated. In step 4 , this new determination is corrected for a possible zero-point offset. This final correction, as well as its origin, are discussed in $\S$ 4.2.2.

At each of the important steps in this process the pipeline produces summary plots that are used to detect possible problems. Information from those quality-check outputs is reported in the final catalog released to the public. In addition, note that the output spectra of the reduction pipeline are in the heliocentric reference frame (see $\S 4.1$ ), and therefore, the RVs provided in the catalog are heliocentric.

\subsubsection{Zero-Point Offset Origin and Correction}

Analysis of raw arc frames taken before and after observations of RAVE fields showed that the positions of the emission lines in one of the arcs are sometimes shifted along the spectral axis on the CCD relative to the other arc. The origin of this shift is most likely to be temperature variations in the spectrograph room, which induce a slight change in some of the optical components. This results in a small offset in the dispersion direction, of the order of a few tenths of a pixel, between the two arc frames. This will also cause an offset between a scientific frame and the arc frame used to calibrate that science frame. The result is a zero-point offset in the measured RVs. A tenth of a pixel corresponds to less than $1.5 \mathrm{~km} \mathrm{~s}^{-1}$, and this is then the order of the zero-point offset.

Both hardware and software were investigated to find the source of the arc shifts. The slit-vane assembly was found to be

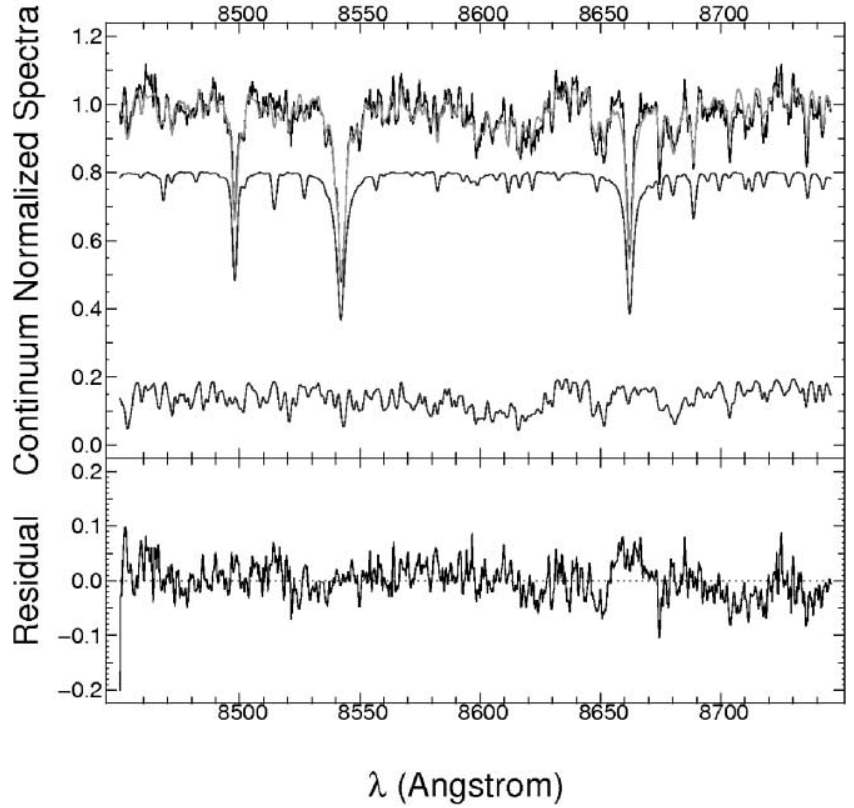

FIG. 7.-Example of the quality of the template fitting that is produced by the algorithm presented in $\S 4.2$, applied to the spectrum of a typical target star, here T6646_01424_1. Top: Observed, continuum-normalized spectrum (black) together with the selected template (gray). The individual first- and second-order components of the template are shown as the second and third lines from the bottom. The second-order contribution for this particular target amounts to less than $\sim 20 \%$. Bottom: Residuals after subtracting the synthetic template from the observed spectrum. [See the electronic edition of the Journal for a color version of this figure.]

vibrationally stable, despite sharing the same mounting on the optical bench as the spectrograph shutter. The spectrograph room temperature is only recorded at the start of a RAVE setup, so the temperature change over the course of a RAVE observation is not known. Figure 8 shows the results of an experiment to test the hypothesis that temperature variations are indeed the source of the shifts. Arc exposures were obtained over the course of a day, with the spectrograph room door shut and the dome shut. The spectrograph room temperature was recorded at the start of each arc exposure. The invar rod temperature was also recorded to check the spectrograph room thermometer. (The invar rod is within the telescope, between the primary mirror and field plate holder. Its temperature is used as an input into a model to maintain focus between the primary mirror and the field plate.) Figure 8 (left) shows that the spectrograph room thermometer is consistent with the invar rod thermometer.

The central fiber of each arc frame was extracted and crosscorrelated with the central fiber of the first arc frame. The crosscorrelation function peak position corresponds to the overall emission-line shift in pixels between each set of arcs. Figure 8 (right) shows a negative pixel shift trend as a result of a positive temperature change, suggesting that the spectrograph room is not a thermally stable environment and that this is responsible for the RV zero-point offset.

If the glass VPH grating were expanding with temperature, the pixel shift would be positive according to the grating equation, implying that this is not the source of the zero-point shift. The thermal expansion coefficient of steel is 1.44 times that of ordinary glass, but steel's thermal conductivity is almost 63 times greater than that of glass. This suggests that various metal components of the spectrograph are temperature-sensitive and thus are responsible for the zero-point shifts. Frequent monitoring of spectrograph temperature in future should improve our understanding of the zero-point shifts. 

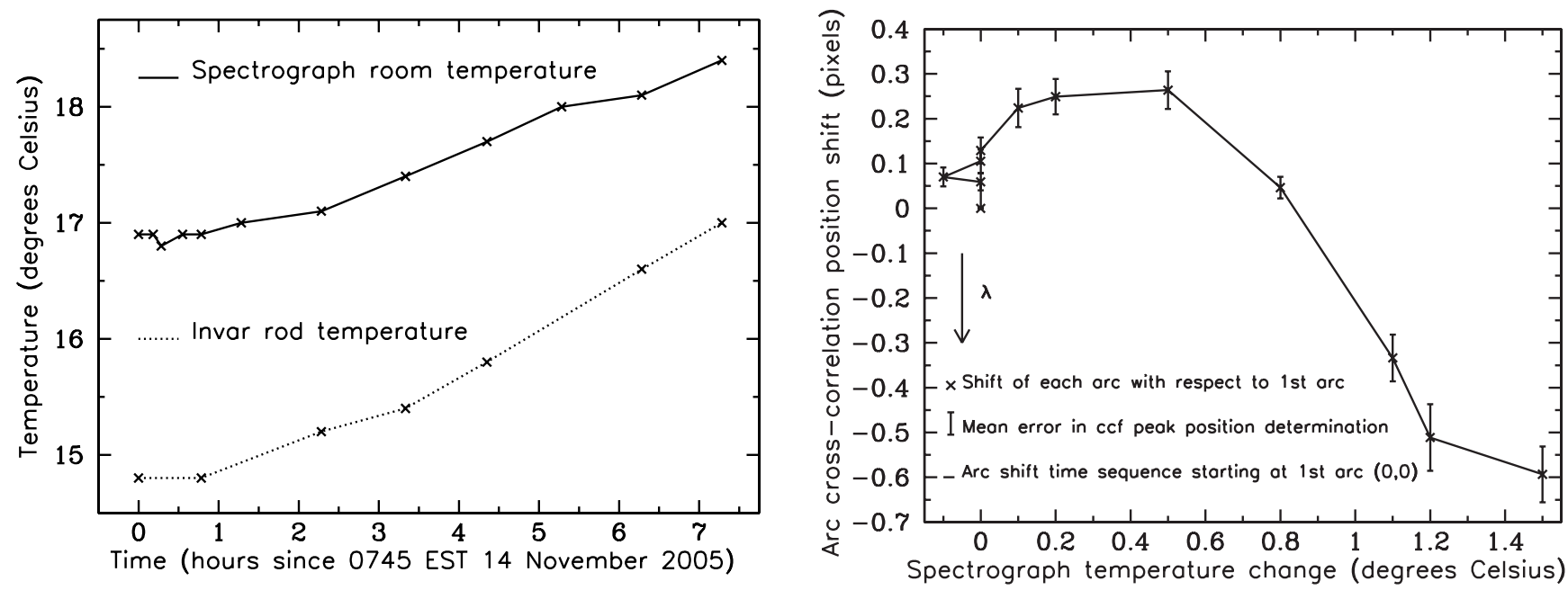

Fig. 8. - Left: Spectrograph room and invar rod temperatures as a function of time. Right: Shift, as a function of temperature, in the position of the cross-correlation peak for the central arc in one frame relative to the central arc in a reference frame (the "first arc").

Given that we do not know the temperature change between a given arc frame and the observation frame to be calibrated with the arc, we need to establish the RV zero-point independent of the arc frames. We therefore developed a procedure using the sky emission lines that are visible in the RAVE wavelength region. We carry out a standard cross-correlation technique with the nonsky-subtracted target frames, again using the median spectrum for each fiber from the set of $\geq 5$ scientific exposures. We use a sky spectrum from the Osterbrock Sky Spectra, ${ }^{29}$ degraded to RAVE's resolution, as a template and compute, for each fiber, the RV associated with the sky in that fiber. As the $\mathrm{S} / \mathrm{N}$ for the sky lines is low, this measurement is precise only to a few kilometers per second (the average RV error for sky spectra is $6 \mathrm{~km} \mathrm{~s}^{-1}$ ). Therefore, a direct correction of a given star's RV using the measurement of the sky zero point in the sky + star spectrum cannot be made. We instead use the fact that across a frame the expected zero-point variation is smooth from fiber to fiber, so that a better zero-point estimation can be obtained by fitting a low-order polynomial to the $\{R V$, Fiber $\}$ data set. Also, in order to give more weight in the fitting procedure to the dedicated sky fibers and to the fibers containing better sky spectra (in the sense that their correlation with the template leads to more accurate velocities), the individual weight follows the Tonry-Davis $R$-value. Further, to reject all unreliable measurements from the fit, fibers with a correlation coefficient lower than 5 are discarded. Finally, the weight for the dedicated sky fibers is doubled, to give an even stronger constraint using the fibers with no stellar spectra. Following this procedure enables us to estimate the zero point to $\sim 1 \mathrm{~km} \mathrm{~s}^{-1}$. An example of this calibration procedure is given in Figure 9. The top panel shows the measured RVs of the sky lines and their associated errors. The thick line is the adopted polynomial fit, and circles indicate the location of dedicated sky fibers. It is clearly seen that pure sky fibers have a much lower error on the determination of the RV, convincing us that more weight must be given to those fibers in the fitting procedure. The bottom panel represents the array of weights used to calculate the polynomial correction for the zero point.

Figure 10 summarizes the statistics of the applied zero-point corrections. The top panel presents their histogram with $0.1 \mathrm{~km} \mathrm{~s}^{-1}$ bins. The bottom panel gives the fraction of RAVE spectra with absolute correction lower than a given value. The absolute value

\footnotetext{
${ }^{29}$ See http://www-mpl.sri.com/NVAO/download/Osterbrock.html.
}

of the applied zero-point correction is lower than $2 \mathrm{~km} \mathrm{~s}^{-1}$ for $80 \%$ of the data.

\section{DATA QUALITY}

\subsection{Signal-to-Noise Ratio and Spectral Resolution}

The $\mathrm{S} / \mathrm{N}$ for a given stellar spectrum was estimated from the scatter of the individual exposures in the sequence of five $10 \mathrm{~min}-$ ute exposures of a given field. At each wavelength the scatter of individual spectra scaled by their mode was determined, omitting the two most discordant points. This scatter then relates to the expected error of the median-combined spectrum. The reported $\mathrm{S} / \mathrm{N}$ per pixel in the final extracted, one-dimensional spectrum is
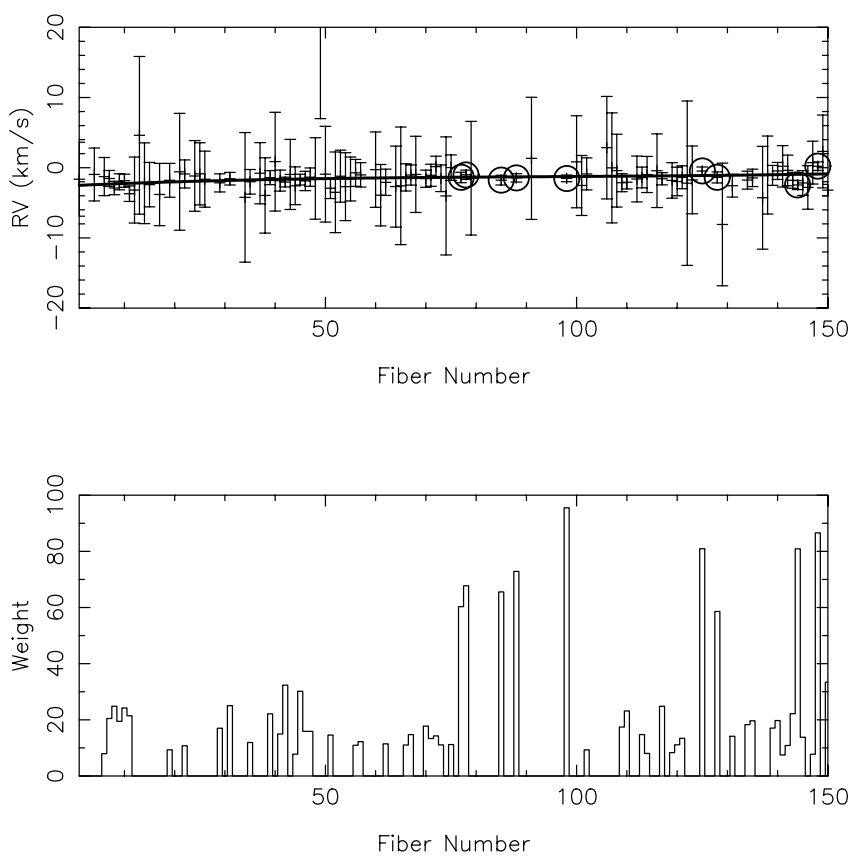

FIG. 9.-Illustration of the procedure for zero-point calibration. Top: Measured RV for the sky lines in each of the spectra in one RAVE field, along with their errors. Circles indicate the location of usable dedicated sky fibers (with an $R$-value above a threshold of 5). Sky fibers with a cross-correlation $R$-value below the limit are discarded from the fit (weight set to 0). Bottom: Weights, proportional to the value of the Tonry-Davis $R$-value, used by the fitting procedure. Usable dedicated sky fibers have their assigned weight doubled. 


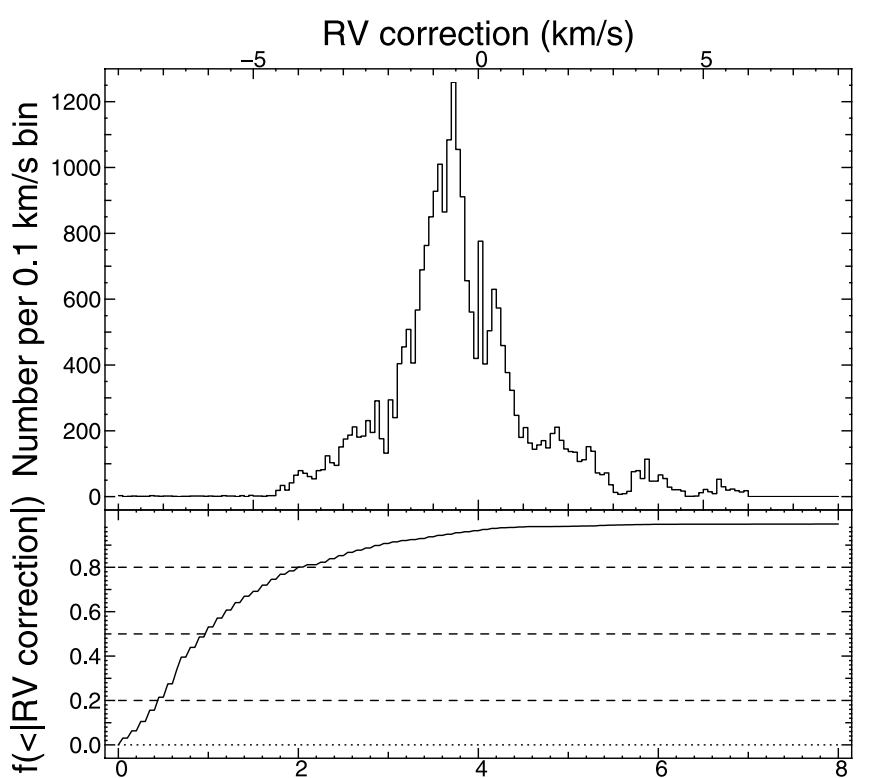

|RV correction| (km/s)

FIG. 10.-Top: Histogram of the distribution of RV correction due to the zeropoint offset. The peak value is at $-0.55 \mathrm{~km} \mathrm{~s}^{-1}$. Bottom: Fraction of RAVE spectra with an absolute value of this correction lower than a given value. Dashed lines show limits at $20 \%, 50 \%$, and $80 \%$.

then calculated as an average over all wavelengths for a given star. Typical counts per pixel of the final one-dimensional spectrum, and per hour of exposure time, for RAVE DR1 follow the approximate relation

$$
N_{\text {counts }}=10^{-0.4\left(I_{\text {DENS }}-20.5\right)},
$$

giving for a complete exposure $\sim 33,000$ counts for an $I=9$ star and $\sim 2000$ counts for an $I=12$ star. Note that these $\mathrm{S} / \mathrm{N}$ measurements, reported in Figure 11, are conservative: the $\mathrm{S} / \mathrm{N}$ in the middle of the spectral domain is generally better than the average, which is what is quoted here. In addition, all calculations were done on spectra that were not normalized. Thus, a varying spectral slope and/or spectral defocusing can also worsen the reported $\mathrm{S} / \mathrm{N}$. We estimate that the accuracy of the reported $\mathrm{S} / \mathrm{N}$ is $\sim 10 \%$.

The resolving power $R=\lambda / \Delta \lambda$ is obtained from measurements of the width of the emission lines in the arc exposures. Near the center of the wavelength range these widths are around 3.1 pixels, which at $\lambda=8600 \AA$ translates into an average resolving power $R \sim 7500$. For stars observed with the first $\sim 30$ fibers, the resolving power is $\sim 25 \%$ lower at the edges of the wavelength range due to spectral defocusing. These values were confirmed by fitting a large set of observed spectra of cool stars with a set of synthetic templates degraded to different spectral resolutions.

\subsection{Radial Velocity Accuracy}

As noted above, the spectra corresponding to the first data release are contaminated by second-order light due to the lack of an OG531 filter. The main effect of this contamination is that it makes it harder to model the spectra, as the amount of contamination is not known a priori and varies both with the spectral type and with time as the blue-to-red transmission of a given fiber changes. The RVs, fortunately, are largely immune to this contamination by second-order light, since the location of the strong absorption lines in the first-order spectra - the primary

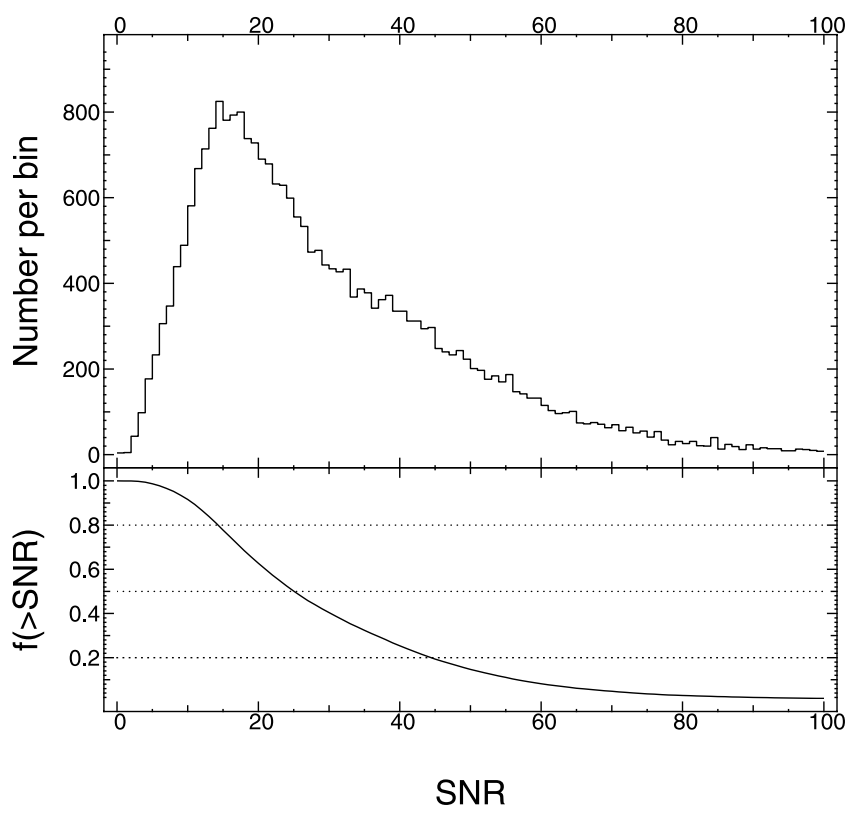

Fig. 11.-Top: Histogram of the distribution of $\mathrm{S} / \mathrm{N}$ per pixel of the final onedimensional spectrum. The peak value is $\mathrm{S} / \mathrm{N} \sim 15$, while the mean value is 29.5 . Bottom: Fraction of RAVE spectra with $\mathrm{S} / \mathrm{N}$ larger than a given value. Dotted lines show limits at $20 \%, 50 \%$, and $80 \%$.

determinations of the wavelength shift in the cross-correlationare not affected. The contamination does lead to a poorer template match than would be achieved for pure first-order spectra, but again, in most cases this results only in a few tenths of a kilometer per second increase in the internal errors (as judged from repeat observations of the same star after the blocking filter was inserted, and hence without the second-order contamination).

The distribution of the internal RV errors is presented in Figure 12. The top panel shows the histogram of the RV error in $0.5 \mathrm{~km} \mathrm{~s}^{-1}$ bins, while the bottom panel is the cumulative

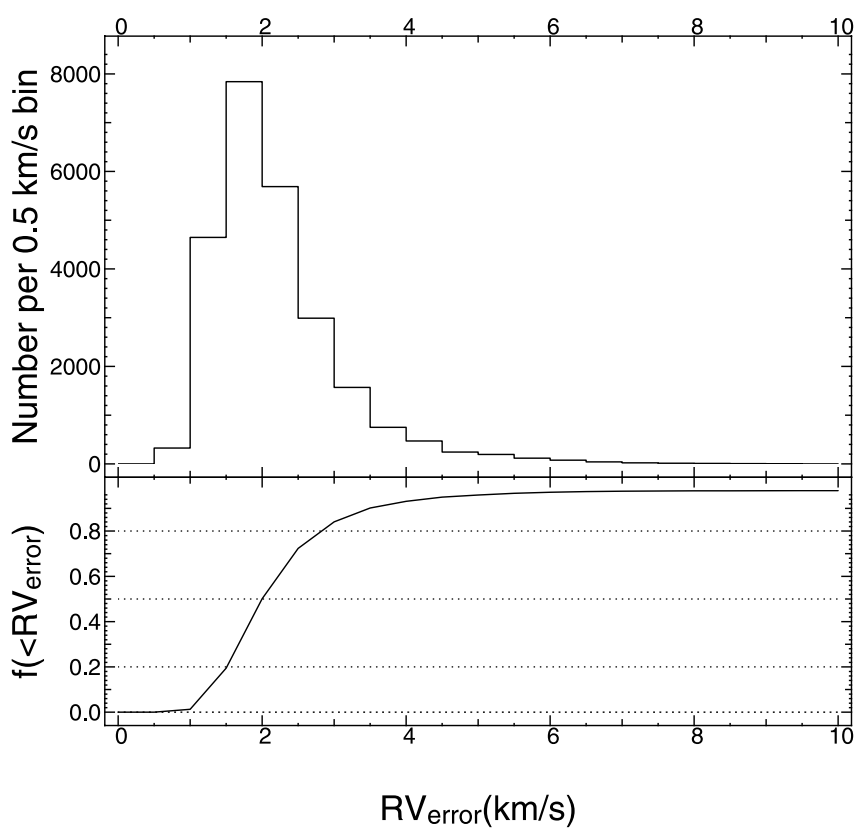

FIG. 12.-Top: Distribution of the internal RV error for RAVE DR1. The peak value is at $1.7 \mathrm{~km} \mathrm{~s}^{-1}$, while the average error for the catalog is $2.3 \mathrm{~km} \mathrm{~s}^{-1}$. Bottom: Fraction of RAVE targets with a RV error lower than a given value. The dotted lines indicate limits of $20 \%, 50 \%$, and $80 \%$. 

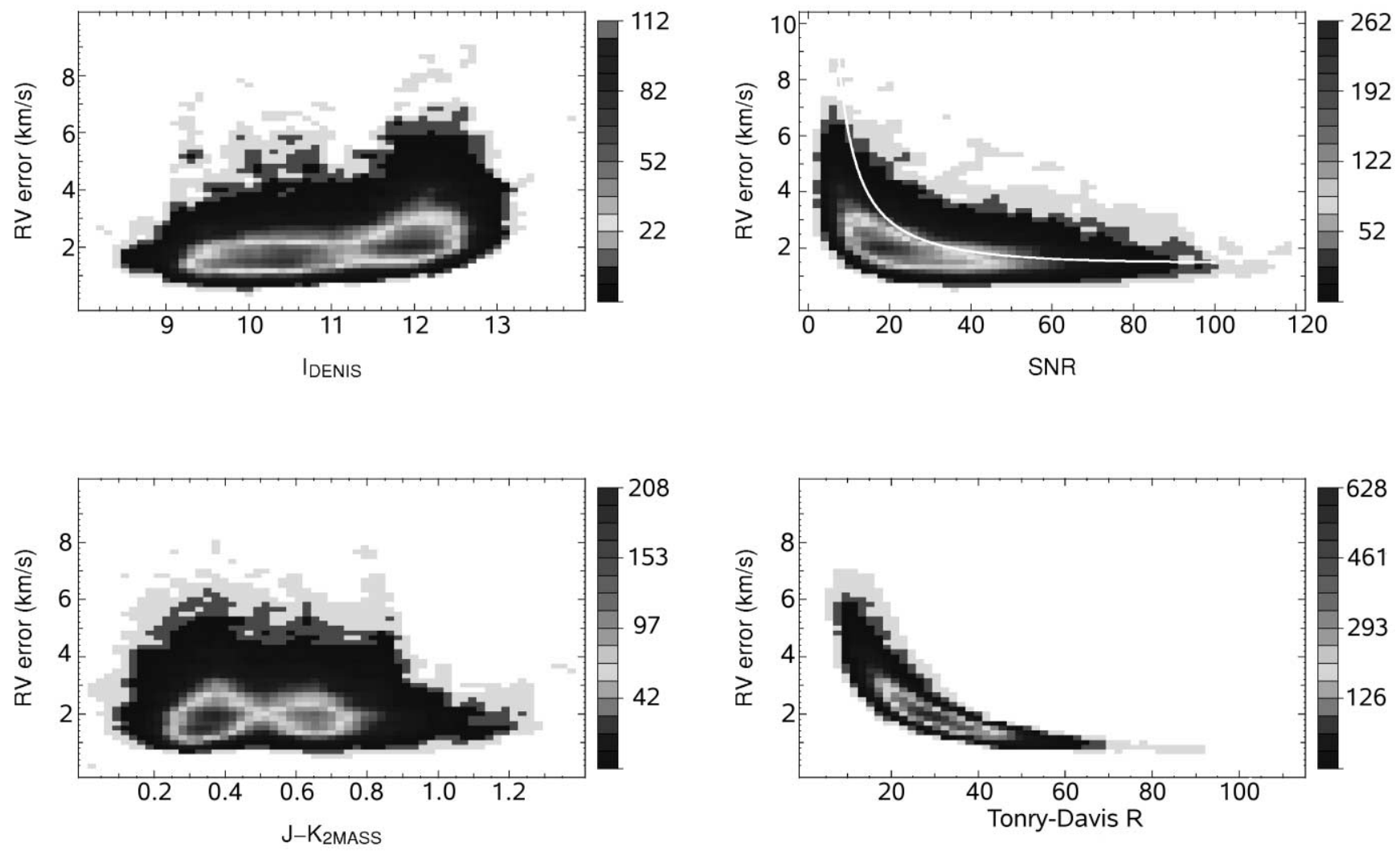

FIG. 13.- Two-dimensional histograms of the distribution of the RV internal error. The shading follows the number of objects per cell, as shown in the keys to the right of the panels. Top left, distribution as a function of $I$ magnitude; top right, $\mathrm{S} / \mathrm{N}$ - the white line follows eq. (4); bottom left, $J-K$ color; bottom right, TonryDavis cross-correlation $R$-value. [See the electronic edition of the Journal for a color version of this figure.]

distribution. The mean internal error is $2.3 \mathrm{~km} \mathrm{~s}^{-1}$, with a peak value of $\sim 1.7 \mathrm{~km} \mathrm{~s}^{-1}$. The bottom panel shows that more than $80 \%$ of RAVE measurements have an internal accuracy better than $3 \mathrm{~km} \mathrm{~s}^{-1}$, and half of the data released reach an accuracy better than $2 \mathrm{~km} \mathrm{~s}^{-1}$.

$\mathrm{RV}$ errors achievable for field stars of $\mathrm{F}-\mathrm{K}$ spectral type have been studied during preparations for the Gaia mission. Munari et al. (2001) made observations of IAU RV standards and derived the following relation for external RV error in kilometers per second as a function of $\mathrm{S} / \mathrm{N}$ and spectral resolving power $R$ :

$$
\begin{aligned}
\log (\mathrm{RV} \text { error })= & 0.6(\log \mathrm{S} / \mathrm{N})^{2}-2.4 \log \mathrm{S} / \mathrm{N} \\
& -1.75 \log R+9.36
\end{aligned}
$$

Its accuracy has been confirmed also by extensive simulations (Zwitter 2002). The error predicted for $R=7500$ and $\mathrm{S} / \mathrm{N}=30$ (typical values for RAVE spectra) is $2.2 \mathrm{~km} \mathrm{~s}^{-1}$. Relation (4) is marked by a white curve in the top right panel in Figure 13. The fact that RAVE performs marginally better than predicted can be traced to several factors: its wavelength range is a bit wider than for Gaia, its sampling is higher than 2 pixels per resolution element, blue-order contamination contributes additional spectral lines carrying velocity information, and our $\mathrm{S} / \mathrm{N}$ estimate is quite conservative.

Figure 13 presents the relation between the RV internal error and various parameters that can influence this accuracy. The top left panel presents the two-dimensional distribution of the RV error as a function of the DENIS $I$-band magnitude. The color coding follows the number of objects per cell from blue (low) to red (high). It can be seen that the accuracy of the velocity determi- nation decreases for stars with fainter $I$-band magnitudes. Nevertheless, there is good consistency between bright and faint targets, with the peak value of the error varying only from $\sim 1.4$ to $\sim 2 \mathrm{~km} \mathrm{~s}^{-1}$ between $I=9$ and 12.5 . The increase in scatter to fainter magnitudes reflects the generally lower $\mathrm{S} / \mathrm{N}$ at these magnitudes, as seen in Figure 14; the average $\mathrm{S} / \mathrm{N}$ is $\sim 10$ for the faintest stars and $\sim 70$ for the brightest ones. It should be noted

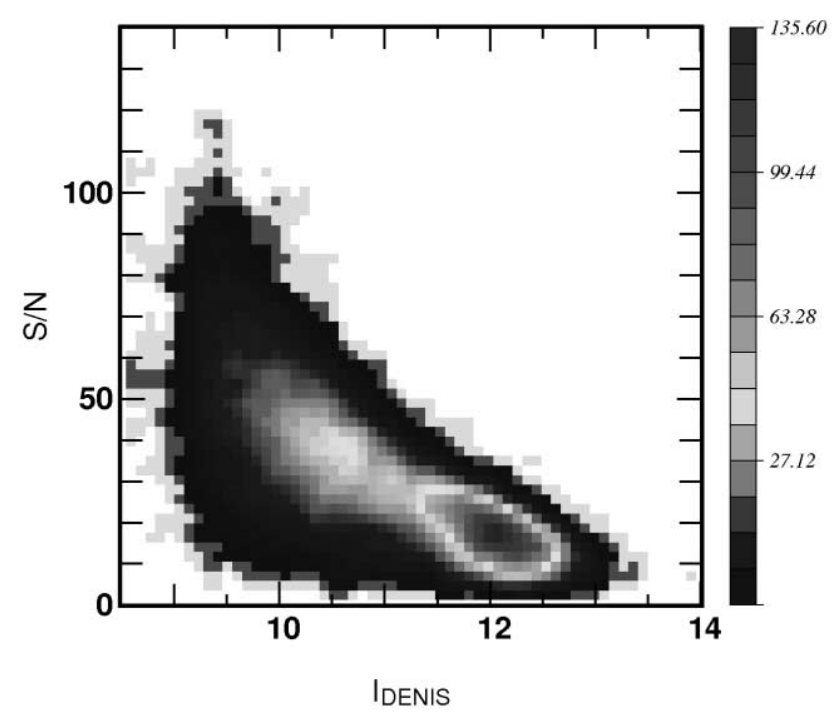

FIG. 14.-S/N vs. DENIS I for RAVE spectra. Again, the shading follows the number of objects per bin, as shown in the key to the right of the panel. [See the electronic edition of the Journal for a color version of this figure.] 
TABLE 1

Description of the Zero-Point Quality Flag

\begin{tabular}{cc}
\hline Flag Value & Dispersion \\
\hline A & $0 \mathrm{~km} \mathrm{~s}^{-1}<\sigma<1 \mathrm{~km} \mathrm{~s}^{-1}$ \\
& $1 \mathrm{~km} \mathrm{~s}^{-1} \leq \sigma<2 \mathrm{~km} \mathrm{~s}^{-1}$ \\
& $2 \mathrm{~km} \mathrm{~s}^{-1} \leq \sigma<3 \mathrm{~km} \mathrm{~s}^{-1}$ \\
& $3 \mathrm{~km} \mathrm{~s}^{-1} \leq \sigma$ \\
& $\ldots \ldots \ldots \ldots \ldots \ldots \ldots \ldots \ldots$ \\
\end{tabular}

that the velocities for the vast majority of the stars, even at the faint limit, remain very accurate.

The effect of Poisson noise is more clearly seen in the top right panel of Figure 13, where the internal RV error is presented as a function of $\mathrm{S} / \mathrm{N}$. This shows that the errors are stable above a threshold value in $\mathrm{S} / \mathrm{N}$ of around 20 , while below this value the velocity errors increase. To quantify the effect, the median value of the internal error, per bin of size 10 in $\mathrm{S} / \mathrm{N}$, varies by only $0.3 \mathrm{~km} \mathrm{~s}^{-1}$ (peak to peak) above $\mathrm{S} / \mathrm{N}=20$, while the lowest two bins are larger by 1.1 and $0.5 \mathrm{~km} \mathrm{~s}^{-1}$. This is also seen in the bottom right panel, as the RV error clearly drops as the crosscorrelation $R$ coefficient decreases. Finally, the bottom left panel shows the distribution of the RV error as a function of the $J-K$ color. No clear variation for the peak value is seen in this diagram, but again, there is some increase in scatter from red to blue. This is due to the emergence of the wide Paschen lines in the spectra of early-type stars and the correspondingly wider correlation function peak.

\subsection{Accuracy of the Zero Point in Radial Velocity}

The discussion above concerns the internal accuracy of the RVs. As mentioned in $\S 4.2 .2$, the RV pipeline also corrects the measurements for zero-point offsets. This correction is computed for each spectrum using a fit to velocities derived from the sky emission lines. This process is, of course, not exact and adds a further error term that must be taken into account when using the RAVE catalog. The combined error (zero-point error + internal error) on the RV is discussed later when comparing RAVE RVs to external data (see $\S 5.6$ ).

The accuracy of the zero-point correction is summarized in the DR1 catalog in the ZeroPointQualityFLAG flag (see Appendix A). This flag is built using a succession of three characters. The first character describes the dispersion between the measured sky RVs and the adopted correction (using the fibers that were used for the fit) for the complete field (therefore a field flag). This character ranges from $\mathrm{A}$ to $\mathrm{E}$, and the corresponding dispersion intervals are reported in Table 1 . As the quality of the fit for an individual spectrum may depend on the location on the CCD, the field is divided into three equal parts according to the fiber number (fibers 1-50, 51-100, and 101-150), and the dispersion is computed for each subgroup offibers (the "Group" flag in Table 2). The second character in the flag summarizes the dispersion for the group to which the fiber belongs (using a flag value from A to D in Table 1). Finally, if the interval between two successive fibers with usable sky velocities is larger than 15 fibers, the zero-point correction may not be well constrained, even without the value of the dispersion being high. For those targets, the last character of the flag is set as an asterisk, indicating a possible zero-point calibration problem.

Table 2 summarizes the fraction of targets with a given flag value for each part of the zero-point correction. Over $97 \%$ of the RAVE targets have a zero-point calibration accurate to better than $2 \mathrm{~km} \mathrm{~s}^{-1}$, and $\sim 73 \%$ are accurate to better than $1 \mathrm{~km} \mathrm{~s}^{-1}$. From these numbers we can conclude that our procedure to cor-
TABLE 2

Summary of Zero-Point Quality Flag Frequency Distributions in RAVE First Data Release for Each Flag Group (See Text)

\begin{tabular}{|c|c|}
\hline Flag Value & Frequency \\
\hline \multicolumn{2}{|l|}{ Field Flag } \\
\hline А & 73.7 \\
\hline В & 23.7 \\
\hline $\mathrm{C}$ & 1.1 \\
\hline 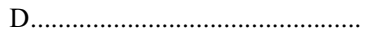 & 0.4 \\
\hline E & 1.1 \\
\hline \multicolumn{2}{|l|}{ Group Flag } \\
\hline$\ldots \ldots \ldots$ & 73.8 \\
\hline В & 23.8 \\
\hline С & 1.8 \\
\hline 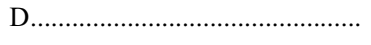 & 0.6 \\
\hline \multicolumn{2}{|c|}{ Possible Bad Calibration } \\
\hline * & 3.7 \\
\hline
\end{tabular}

rect from zero-point offset is efficient, enabling us to keep the zero-point error term below $2 \mathrm{~km} \mathrm{~s}^{-1}$ for the vast majority of our targets.

Adopting a zero-point error of $1 \mathrm{~km} \mathrm{~s}^{-1}$, the mode of the $\mathrm{RV}$ error distribution for the RAVE DR1 catalog is $2 \mathrm{~km} \mathrm{~s}^{-1}$.

\subsection{Repeated Observations}

A total of 428 stars in the present data release were observed more than once during the first year of operation. Of these, 98 stars were observed three times.

Assuming that any variation of the measured RVs is not intrinsic, one can thus estimate the accuracy of the catalog. The distribution of the derived RVs of the repeat observations is shown in Figure 15. The mean deviation is essentially zero, and the rms

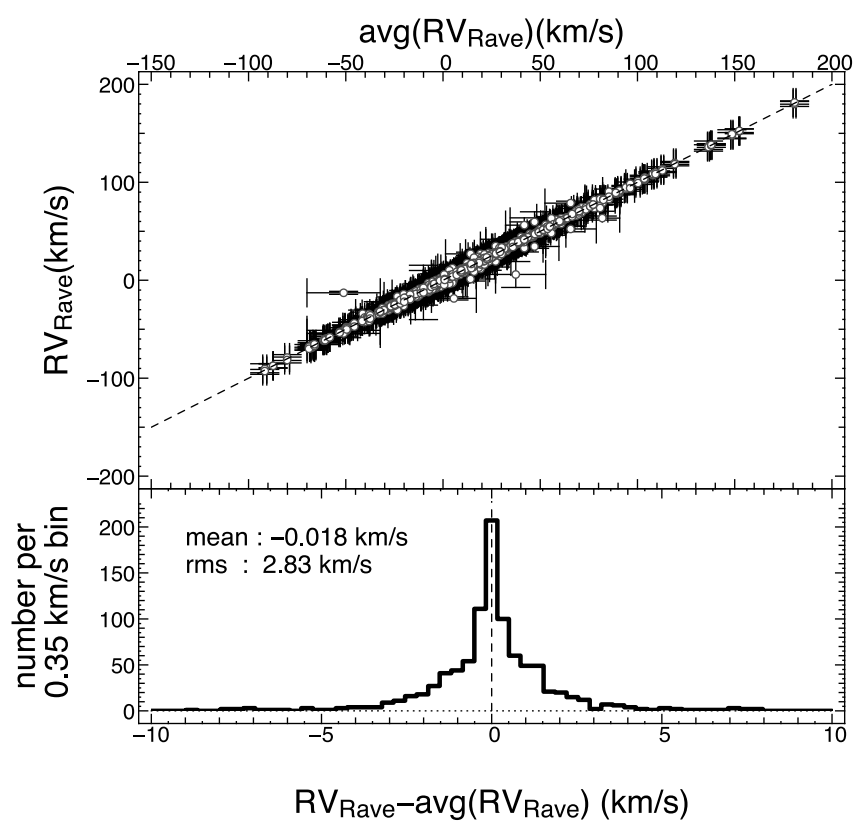

FIG. 15.- RV accuracy checked by repeated observations of 428 stars. The scatter of $2.83 \mathrm{~km} \mathrm{~s}^{-1}$ is consistent with estimates of internal and zero-point $\mathrm{RV}$ errors. [See the electronic edition of the Journal for a color version of this figure.] 
of $2.83 \mathrm{~km} \mathrm{~s}^{-1}$ is perfectly consistent with the internal error and zero-point accuracy given above.

\subsection{Contamination by Binary Stars}

Most of the stars in the present data release are observed only once, so we have no way of knowing whether a given star is single or whether the observed RV may in part reflect the instantaneous radial component of the orbital motion in a binary system. The average orbital velocity of star 1 in orbit around star 2 is

$$
\left\langle V_{\text {orb }}\right\rangle=v_{o} q(1+q)^{-1}(M / P)^{1 / 3},
$$

where $v_{o}=30 \mathrm{~km} \mathrm{~s}^{-1}, q=M_{2} / M_{1}$ is the mass ratio, and $M$ and $P$ are the total mass and orbital period, expressed in solar masses and years for the resulting velocity in kilometers per second. Thus, for most single-lined systems, with an orbital period of years or less, the orbital RV projection exceeds $5 \mathrm{~km} \mathrm{~s}^{-1}$, and their kinematic data may be inaccurate and even potentially misleading. This is consistent with the $15 \mathrm{~km} \mathrm{~s}^{-1}$ value for the average scatter of individual RVs of spectroscopic binaries found by Nordström et al. (2004). Note that these authors made many repeat observations and identified $19 \%$ of their sample as spectroscopic binaries of some kind. Double-lined systems show asymmetric or even double-peaked shapes of the correlation function. The present release contains 27 targets flagged as possible binaries, since they show clear asymmetric profiles for the correlation function, as well as a poor template match or clear double-line spectra. Nevertheless, we cannot unequivocably state that these are binaries without temporal orbital variations to the RVs, as nonintrinsic effects (for example, scattered-light residual or fiber cross talk) could also contribute. Therefore, this information should be used with caution.

Photometric variability is an efficient way to discover binary systems with periods of hours or days (Piquard et al. 2001), while systems with periods of hundreds of years are spatially resolved (see Figs. 3 and 4 from Zwitter \& Munari 2004). Unfortunately, no repeated photometric observation or light curves are available for stars in this data release. A cross-check with the general catalog of variable stars ${ }^{30}$ revealed 22 matches. Those objects are flagged in the catalog using the VarFLAG entry (see Appendix A). While every effort has been made to identify visual binaries from available catalogs, as well as at the telescope, we note that the amplitudes of the orbital velocities of member stars of such systems are very small, and indeed less than our internal accuracy, so would not affect our quoted RV values.

In future years repeats will be obtained for a larger fraction of stars, including some of the stars from this data release. This will help to identify spectroscopic binaries and active stars.

\subsection{Comparison with External Data Sets}

To further check the consistency of our RVs, we compare our measurements with external data from three main sources: ${ }^{31}$ dedicated observations using the ELODIE spectrograph, dedicated observations using the Mount Stromlo and Siding Spring Observatories (MSSSO) $2.3 \mathrm{~m}$ telescope, and overlap with the sample of the GCS. Each subset will be briefly described below.

ELODIE data.-ELODIE is a cross-dispersed echelle spectrograph mounted at the Cassegrain focus of the $1.93 \mathrm{~m}$ telescope at the Observatoire de Haute Provence. The spectra cover

\footnotetext{
${ }^{30}$ VizieR Online Data Catalog, 2250 (N. N. Samus et al., 2001).

31 A few stars from this release were also observed with the echelle spectrograph at the Apache Point Observatory $3.5 \mathrm{~m}$ telescope as part of a follow-up scientific project. They will be published as part of the science paper.
}

a $3000 \AA$ Aavelength range (3850-6800 A) with a spectral resolution of 42,000 . The instrument is entirely computer-controlled, and a standard reduction software package (TACOS) automatically processes the data. Seven stars of this first release, selected simply to be bright and accessible, were observed on three nights in 2004 July (July 5, 8, and 9). The $\mathrm{S} / \mathrm{Ns}$ of the spectra range from 1 to 5 pixel $^{-1}$ (10-20 minutes of exposure time), sufficient at these high spectral resolutions. ${ }^{32}$ We cross-correlated the spectra against the most appropriate spectral template given by the ELODIE reduction pipeline. ${ }^{33}$ The results are given in Table 8 . The limiting accuracy ranges from $300 \mathrm{~m} \mathrm{~s}^{-1}$ to $1 \mathrm{~km} \mathrm{~s}^{-1}$, with the exception of the probable double star (TYC 50314781 ), which has been discarded from the analysis. From the six single stars measured with ELODIE for this release, we obtained a dispersion $\sigma\left(\mathrm{RV}_{\text {ELODIE }}-\mathrm{RV}_{\mathrm{RAVE}}\right)=1.7 \mathrm{~km} \mathrm{~s}^{-1}$, dominated by errors in the RAVE measurements. We find a negligible offset, with the mean being $\left\langle\left(\mathrm{RV}_{\text {ELODIE }}-\mathrm{RV}_{\mathrm{RAVE}}\right)\right\rangle=-0.1 \pm 0.8 \mathrm{~km} \mathrm{~s}^{-1}$, showing that the final zero-point error for RAVE data is much smaller than the measurement dispersion.

$2.3 \mathrm{~m}$ data.-Long-slit spectra were taken with the Double Beam Spectrograph (Rodgers et al. 1988) on the MSSSO $2.3 \mathrm{~m}$ telescope, over a period of 2 yr from 2003 December to 2005 September. The average seeing was $1.5-2.5$, where a narrow slit (1".5) minimized slit positioning errors. The spectral resolution is similar to RAVE $(R \sim 8000)$ with $0.55 \AA$ pixel $^{-1}$. The stars, randomly selected from the first year of observation with the exception of a few high-velocity candidates, were observed at a vertical angle of $0^{\circ}$ or $180^{\circ}$ to eliminate atmospheric dispersion effects. The average $\mathrm{S} / \mathrm{N}$ was $\sim 90$ pixel $^{-1}$. We cross-correlated these spectra against a subset of the Zwitter et al. (2004) spectra using XCSAO in IRAF. The best match was chosen as the template with the highest $R$ coefficient ( Tonry \& Davis 1979). Only those stars with a best-match $R>40$ were used - this limit was found empirically to minimize errors in $2.3 \mathrm{~m} \mathrm{RVs}$. The resulting internal error of the $2.3 \mathrm{~m}$ RVs from these methods, measured against high-precision Nordström et al. (2004) stars and RV standards, is $\sim 1.5 \mathrm{~km} \mathrm{~s}^{-1}$. The results are given in Table 9 .

The 77 stars belonging to this data release and measured with the $2.3 \mathrm{~m}$ telescope cover a $\mathrm{RV}$ range of $820 \mathrm{~km} \mathrm{~s}^{-1}$. Of those, we believe seven outliers are likely single-lined spectroscopic binaries, as the difference in RV between the RAVE and $2.3 \mathrm{~m}$ measurements deviates by over $3 \sigma$. Excluding these seven outliers, we find $\sigma\left(\mathrm{RV}_{2.3 \mathrm{~m}}-\mathrm{RV}_{\mathrm{RAVE}}\right)=3.4 \mathrm{~km} \mathrm{~s}^{-1}$ and $\mu\left(\mathrm{RV}_{2.3 \mathrm{~m}}-\right.$ $\left.\mathrm{RV}_{\mathrm{RAVE}}\right)=0.1 \pm 0.4 \mathrm{~km} \mathrm{~s}^{-1}$. Correcting for $2.3 \mathrm{~m}$ internal error, we conclude from this sample that the RAVE RV error is $\sigma=$ $3.0 \mathrm{~km} \mathrm{~s}^{-1}$, with a negligible mean offset.

Geneva-Copenhagen targets.-DR1 targets have been crossmatched with the GCS catalog, which by virtue of many repeated observations contains binarity indicators in addition to accurate RVs. We found 13 matches, two of them classified as binaries in the GCS.

The resulting 11 "single" targets show acceptable agreement, with a mean difference of $1.4 \pm 0.4 \mathrm{~km} \mathrm{~s}^{-1}$ and an rms of $1.4 \mathrm{~km} \mathrm{~s}^{-1}$. The results are given in Table 10 .

Figures 16-19 summarize the comparison of RAVE RVs with the external data. Figure 16 shows the location in the 2MASS color-color diagram of the RAVE targets with external measurements. The broad distribution of those objects in this diagram indicates that we have good coverage of the global stellar properties,

\footnotetext{
32 An accuracy of $10 \mathrm{~m} \mathrm{~s}^{-1}$ can be reached for planet searches using this instrument.

${ }^{33}$ TACOS provides two templates: the first one, $\mathrm{R} 37000 \mathrm{~K} 0$, is valid for $\mathrm{G}, \mathrm{K}$, and $\mathrm{M}$ stars, while R37000F0 is used for spectral types around F0.
} 


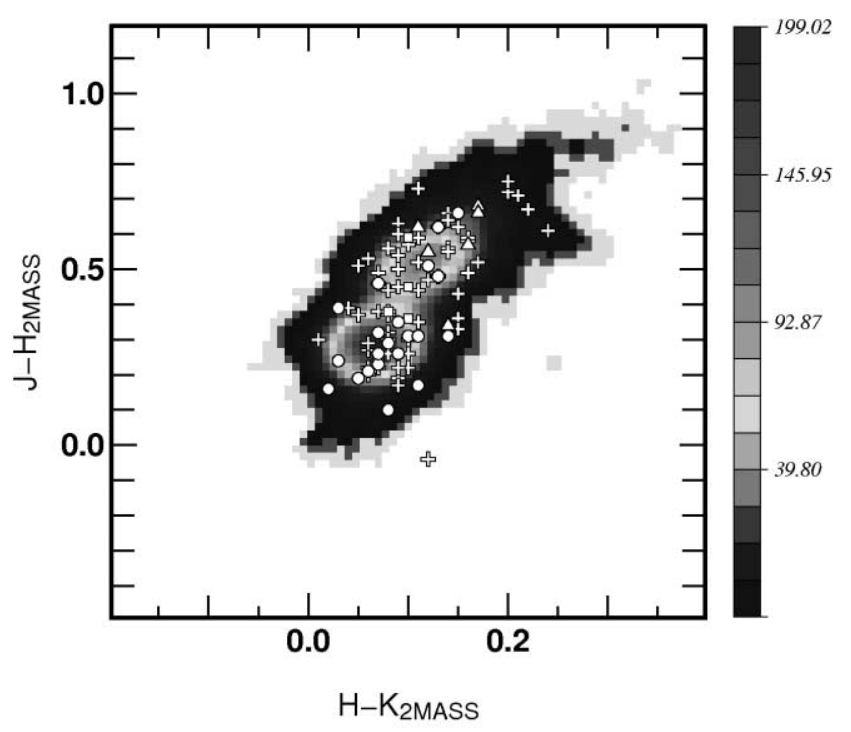

FIG. 16.- Infrared color-color diagram of the stars in DR1. The locations of our targets with external data are overplotted using white symbols. Crosses are for $2.3 \mathrm{~m}$ data, triangles for ELODIE targets, and squares for GCS targets. Circles denote the location of binary targets. The external targets are well distributed across this parameter space, indicating that our reference target selection is not biased toward any particular type of object. [See the electronic edition of the Journal for a color version of this figure.]

with the exception only of the reddest giants $(H-K \gtrsim 0.2)$. Our external checks should therefore be applicable to the full survey, despite the low number of external targets.

Figure 17 presents the comparison between the RAVE RVs and the external measurements. The top panel shows the RV-to$\mathrm{RV}$ comparison, with each different source plotted with different symbols (open circles are for binary systems that are removed

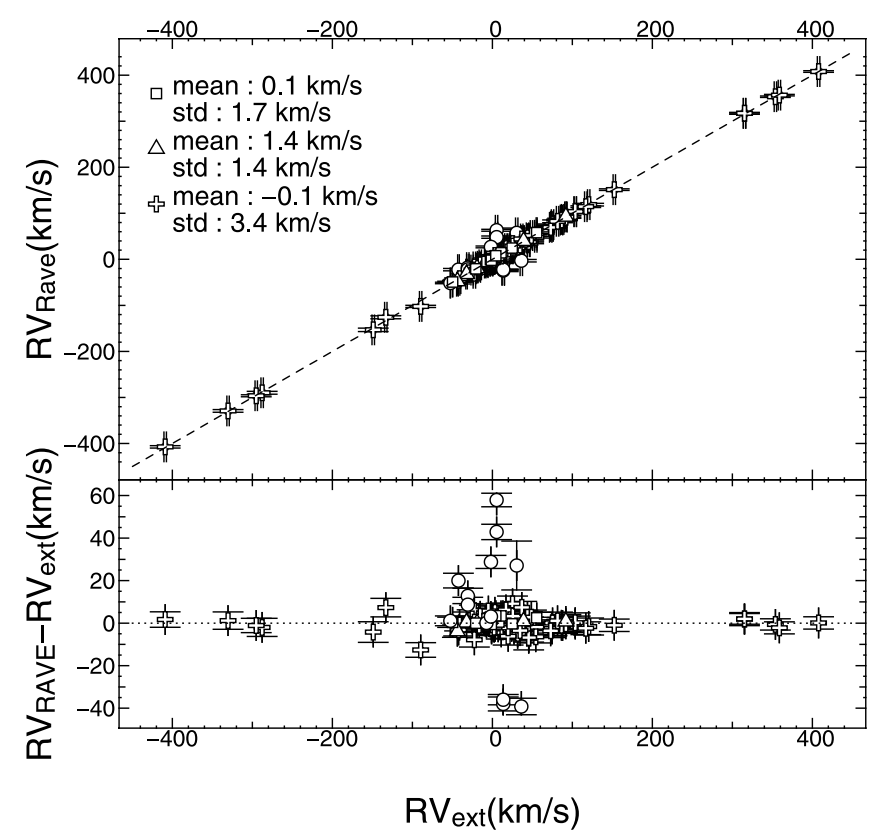

FIG. 17.-Comparison of RAVE RVs with external measurements. The top panel presents the external to RAVE RV comparison. The bottom panel shows the RV difference as a function of RV for the three different reference groups. Squares represent ELODIE targets, triangles represent GCS targets, and crosses represent $2.3 \mathrm{~m}$ targets. Circles denote suspected and confirmed binary objects and are removed from the analysis. The mean difference and standard deviation are reported for each group in the top panel. RAVE RVs show a good agreement with independent measurements.

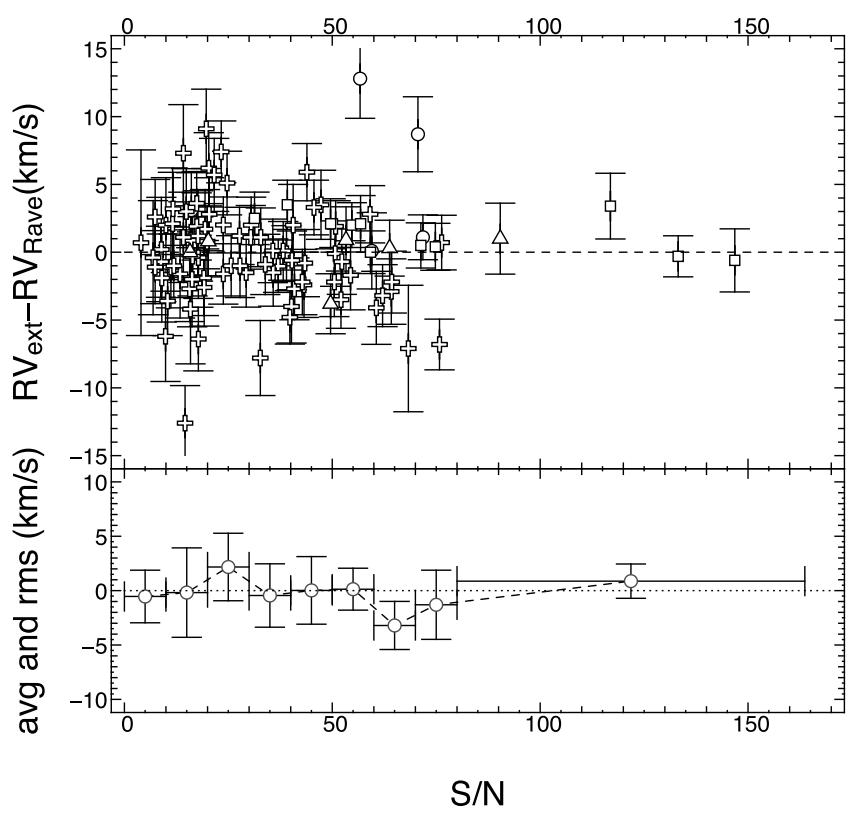

Fig. 18.-Behavior of the RV difference with S/N. Top: RV difference as a function of $\mathrm{S} / \mathrm{N}$ for each group of external measurements. Symbols are the same as in Fig. 16. Bottom: Average deviation and rms as a function of $\mathrm{S} / \mathrm{N}$ for the sum of all external measurements. This plot shows no apparent bias as a function of $\mathrm{S} / \mathrm{N}$, the mean difference being consistent with zero for all values of $\mathrm{S} / \mathrm{N}$. One bin $(60<\mathrm{S} / \mathrm{N}<70)$ has a rather large deviation due to a single object with large error. [See the electronic edition of the Journal for a color version of this figure.]

from the analysis). For each data source, the mean difference and standard deviation are also given. As the spread in RV is large, the bottom panel gives the RV difference as a function of RV. This plot clearly shows that the binary systems produce a large offset, while single stars show good agreement, with close to zero velocity difference. We conclude in $\S 5.3$ that the mode is $2 \mathrm{~km} \mathrm{~s}^{-1}$, the median RAVE error being $\sim 2.2 \mathrm{~km} \mathrm{~s}^{-1}$. Combining the measurements from all three samples, the mean difference

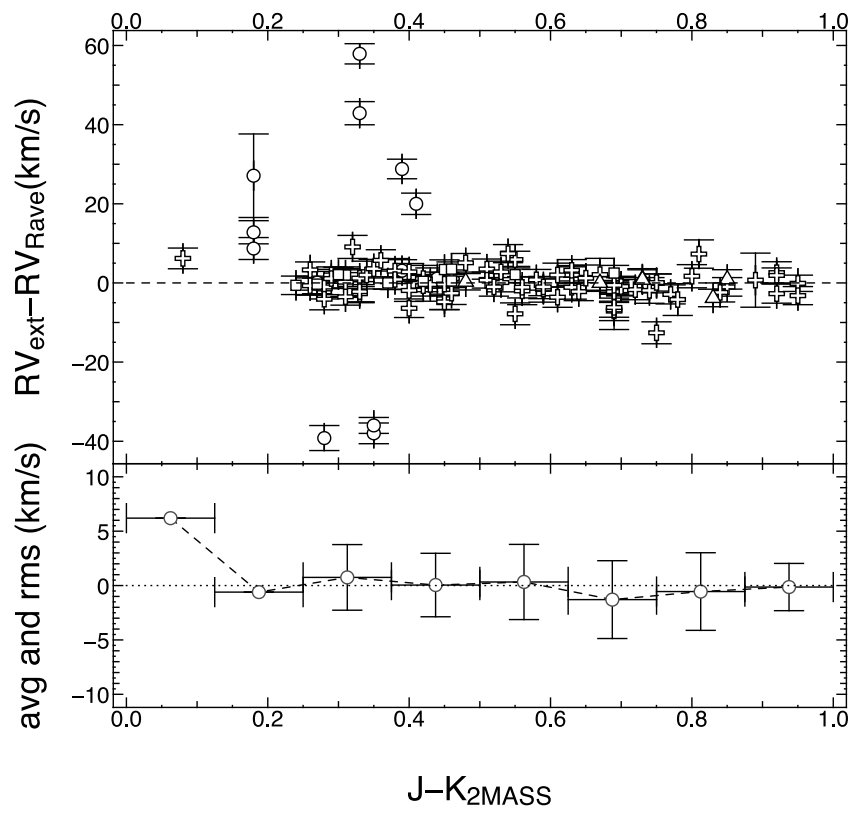

FIG. 19.-Behavior of the RV difference with $J-K$ color from the 2MASS catalog. Arrangement and symbols are the same as in Fig. 18. RV errors do not depend on the color of the target except for the bluest targets. [See the electronic edition of the Journal for a color version of this figure.] 


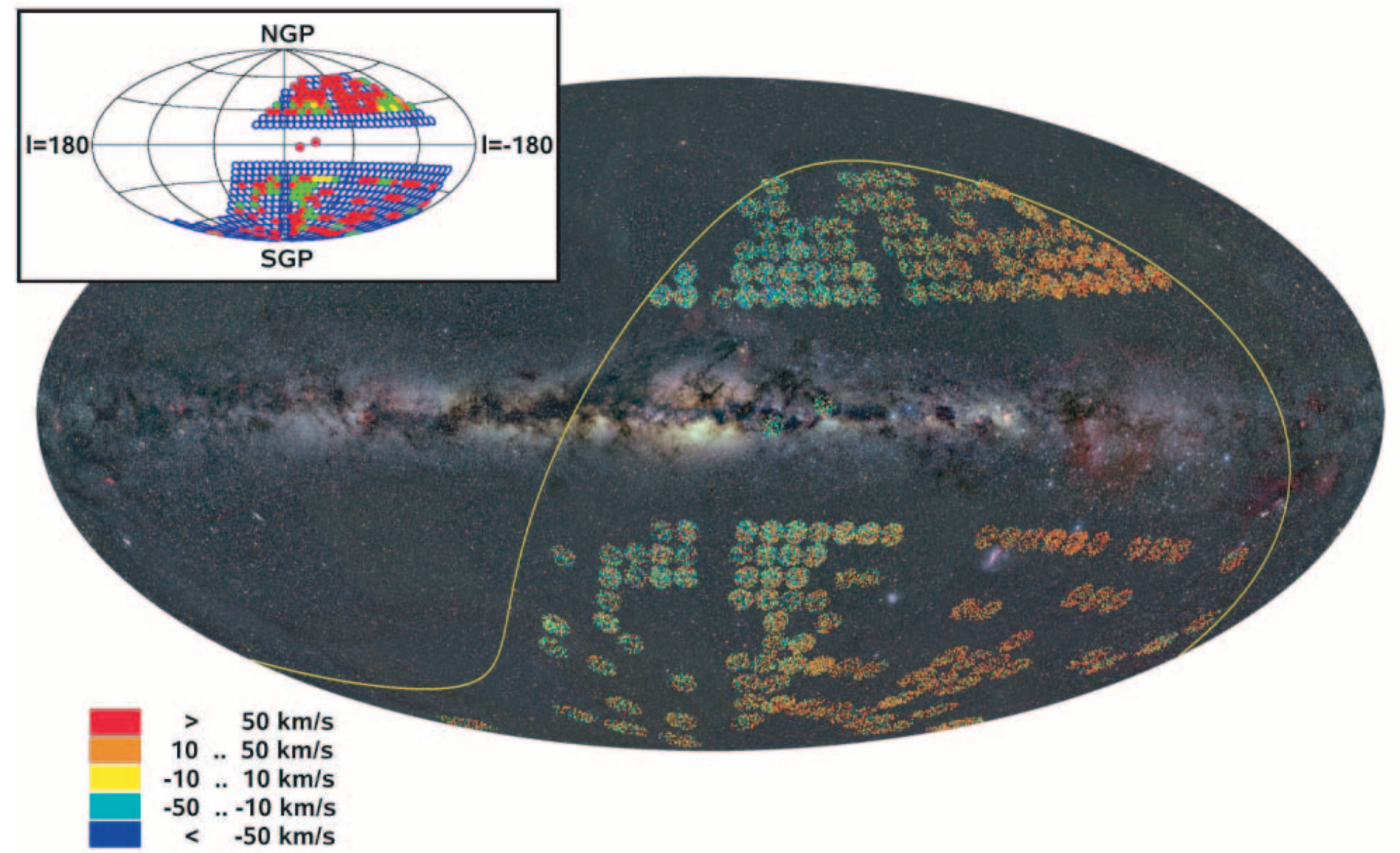

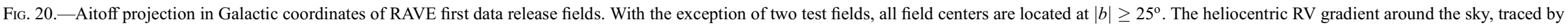

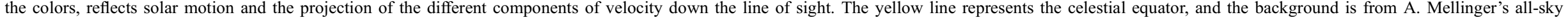

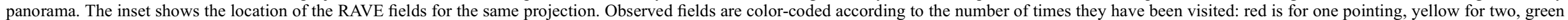
for three, and light brown for four. 
TABLE 3

Number and Fraction of RAVE Targets with a Counterpart in the Photometric Catalogs

\begin{tabular}{|c|c|c|c|c|c|c|}
\hline \multirow[b]{2}{*}{ Catalog Name } & \multirow{2}{*}{$\begin{array}{l}\text { Number of } \\
\text { ObJects }\end{array}$} & \multirow{2}{*}{$\begin{array}{l}\text { Percentage of DR1 } \\
\text { with CounterPart }\end{array}$} & \multicolumn{4}{|c|}{ Percentage with Quality Flag } \\
\hline & & & A & B & $\mathrm{C}$ & $\mathrm{D}$ \\
\hline 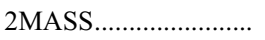 & 25,268 & 99.98 & 99.6 & 0 & 0 & 0.4 \\
\hline DENIS ........................... & 18,637 & 73.7 & 75.3 & 22.3 & 1.8 & 0.6 \\
\hline USNO-B ......................... & 24,814 & 98.2 & 99.2 & 0.5 & 0 & 0.3 \\
\hline
\end{tabular}

between RAVE RVs and external measurements is $-0.04 \pm$ $0.29 \mathrm{~km} \mathrm{~s}^{-1}$ with a standard deviation of $3 \mathrm{~km} \mathrm{~s}^{-1} .^{34}$ This is in good agreement with our predicted accuracy.

As a further test, we compare in Figure 18 the RV difference to the $\mathrm{S} / \mathrm{N}$ in RAVE data. The top panel in this figure is a simple scatter plot including all available data, while the bottom panel presents the mean difference and rms for intervals of 10 in $\mathrm{S} / \mathrm{N}$. The offset in the interval $50 \leq \mathrm{S} / \mathrm{N}<60$ is due to a single measurement with large error and $R V$ difference $\left(\mathrm{RV}_{\text {diff }}=7 \pm 5 \mathrm{~km} \mathrm{~s}^{-1}\right)$. This figure shows no dependence of the RV on $\mathrm{S} / \mathrm{N}$, the mean and rms being consistent with no trend. Again, the amplitude of the scatter increases as the $\mathrm{S} / \mathrm{N}$ drops below $\sim 20$, as expected from the top right panel of Figure 13.

Finally, Figure 19 demonstrates that RV errors do not depend on color. The exception is the bluest of our targets $(J-K<$ 0.1 , which are dominated by wide hydrogen lines with only a handful of weak metallic lines.

In summary, a comparison of the RAVE velocities with those from the three external sources (ELODIE, GCS, and $2.3 \mathrm{~m}$ ) reveals that the offset in mean velocity is very small, most likely less than $1 \mathrm{~km} \mathrm{~s}^{-1}$. The errors in the RAVE stars in common with the ELODIE and GCS observations are 1.7 and $1.4 \mathrm{~km} \mathrm{~s}^{-1}$, respectively, while the corresponding errors in the RAVE velocities of $2.3 \mathrm{~m}$ stars are larger, about $3.0 \mathrm{~km} \mathrm{~s}^{-1}$. We note that the ELODIE and GCS stars are significantly brighter in the mean than the $2.3 \mathrm{~m}$ stars (see Fig. 5). However, Figure 18 shows that the difference in estimated errors is probably not due to different $\mathrm{S} / \mathrm{N}$ values for the RAVE spectra. A more likely explanation is that the contamination from stars in adjacent fibers, while small, will contribute more to the velocity errors for the fainter $2.3 \mathrm{~m}$ stars.

\section{FIRST DATA RELEASE}

\subsection{Spatial Coverage and Global Properties}

The first RAVE data release presents RVs for 24,748 apparently bright stars in the Milky Way. The total number of spectra collected for these stars is 25,274 (including reobservations). Those spectra were obtained during 67 nights between 2003 April 11 and 2004 April 2, with the exception of one field observed on 2004 August 3 (second year, but affected by secondorder contamination). The 12,836 stars in this release are Tycho- 2 entries, and 11,921 are extracted from the SSS. The stars are distributed over $2356 \mathrm{dF}$ fields (for 240 observed including reobservations) for a total area covered of $\sim 4760 \mathrm{deg}^{2}$.

The locations on the sky of the RAVE fields of DR1 are indicated in Figure 20. This figure presents an Aitoff projection of all RAVE DR 1 target fields in Galactic coordinates. Each circle is a RAVE target field ( $5^{\circ} .7$ in diameter). Filled fields are part of this data release. The color coding indicates the number of pointings for a given field (remember that each field contains up to three sets of targets): red for one time, yellow for two, and green

\footnotetext{
${ }^{34}$ The standard deviation corrected for external source mean error is $\sigma=2.7 \mathrm{~km} \mathrm{~s}^{-1}$
}

for three. Among those, four fields are reobserved with the same set of targets (three fields have been reobserved a second time, one field three times). In addition to the normal RAVE target fields, which are restricted to $|b|>20^{\circ}$, two fields observed to test the MK classification scheme in the red RAVE wavelength region are located closer to the Galactic plane.

\subsection{Photometry}

As noted earlier, the photometry of the input catalog was not homogeneous: the bright part of the sample was selected using a pseudo- $I$ criterion based on Tycho- $2 V_{\mathrm{T}}$ and $B_{\mathrm{T}}$ magnitudes, while the faint part was selected from SuperCOSMOS $I$ data (see $\S 2.3$ ). To maximize the usability of the DR 1 data, cross identification with optical and near-infrared catalogs (USNO-B, DENIS, and 2MASS) is provided. The matches are selected using a nearestneighbor association. A large search radius is used to check for possible contamination, given that the diameter of a $6 \mathrm{dF}$ fiber on the sky is 6.7 .

In addition to the distance to the nearest neighbor, a quality flag summarizing the reliability of the association is provided in the catalog. This single-character flag is set to A for clear association, meaning that the distance between the target and the nearest neighbor is less than $1^{\prime \prime}$, with no other possible association. The flag value B (or C) is used to warn that two (or more) associations are found within a $1^{\prime \prime}$ radius, and $\mathrm{D}$ indicates that the nearest neighbor is further away than $2^{\prime \prime}$. A value $\mathrm{X}$ is given for the flag when no association is found. Table 3 summarizes the outcome of the cross-identification procedure for the various photometric catalogs.

For 2MASS and USNO-B, the cross identification appears well defined. The fraction of objects with a flag value different from $\mathrm{A}$ is lower than $1 \%$, indicating good agreement between the astrometry in the RAVE input catalog and USNO-B. We therefore expect the level of false identification to be lower than $1 \%$ for those catalogs.

The result of the cross identification with DENIS appears worse than for 2MASS or USNO-B. The main differences are in the categories $\mathrm{B}$ and $\mathrm{C}$, where multiple matches are found. This is due to problems in the DENIS catalog by which, at the edges of the detector, the astrometry and photometry both become less accurate. In this case, DENIS reports multiple detections of the object for the different scans. Those detections have almost identical magnitudes and positions but could not be associated with the same object. In all those cases, we use the nearest neighbor. As the difference in magnitude is small between the possible matches, this should not affect the overall quality of DENIS associations. In addition, the reported magnitudes should be of sufficient accuracy for most of the uses of the RAVE catalog. Therefore, even if the cross identification with the DENIS catalog seems to be poorer according to Table 3, it does not significantly lower the validity of the cross-identification.

The resulting $I$-band distribution for the RAVE catalog is given in Figure 21 using the cross identification with DENIS. 


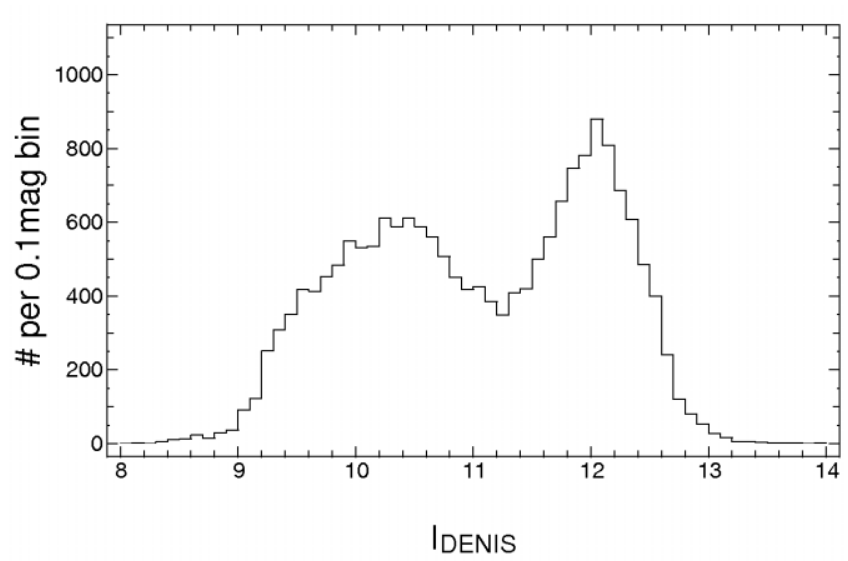

FIG. 21.-DENIS I-band apparent magnitude distribution of the RAVE DR1 catalog. The two peaks in this distribution are the result of our selection criteria in the pseudo- $I$ band and delimit the bright and faint samples.

This luminosity function exhibits two peaks, defining the bright and faint samples: the first one is centered on $I=10.2$, the second one on $I=12$. The shape of this function clearly indicates that, at this point, RAVE does not approximate a random magnitudelimited sample. As discussed in $\S 2.3$, this is an effect of the input catalog being selected either using the pseudo- $I$ magnitude derived from Tycho-2 photometry or using SuperCOSMOS $I$ in the bright regime, where systematic offsets are to be expected. This effect will be corrected in future RAVE releases with the availability of the full DENIS catalog, which will be used for the input catalog of later data releases.

Figure 22 presents the 2MASS color-color diagram of the data release. Fiducial colors from Table 2 of Wainscoat et al. (1992) have been added for clarity, with dark and light curves representing dwarf and giant stars, respectively. This clearly shows that, as intended, the data release probes both the nearby and more distant Galaxy. As an example, K0 dwarfs in the RAVE catalog span an approximate distance range of $\sim 50$ to $\sim 250 \mathrm{pc}$, while $\mathrm{K} 0$ giants are located in the distance range $700 \mathrm{pc}$ to $3 \mathrm{kpc}$.

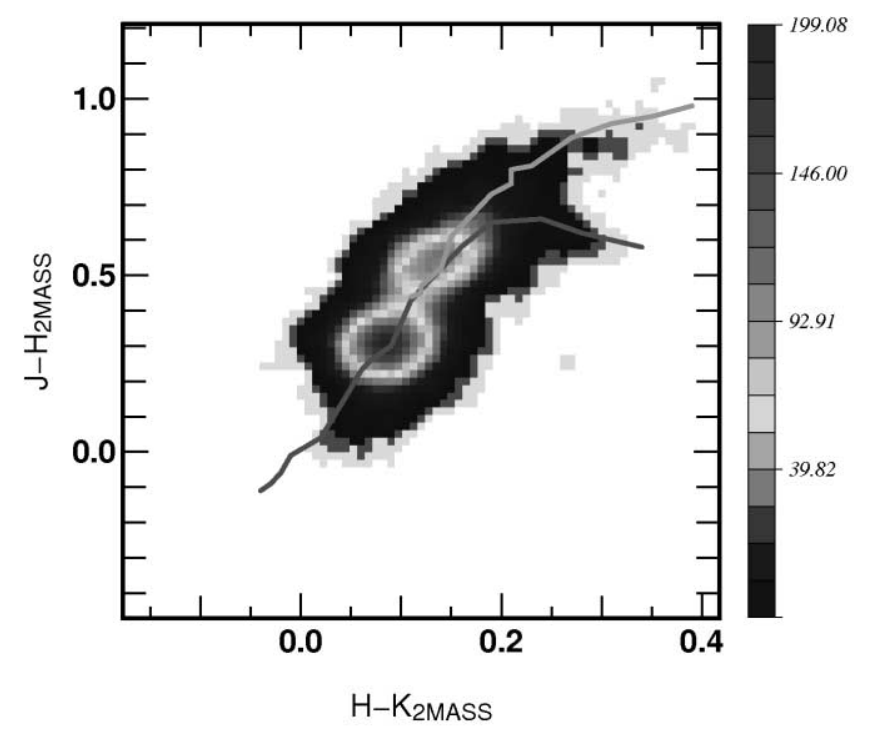

FIG. 22.-2MASS infrared color-color diagram for RAVE targets in the first data release. For clarity, the predicted loci for dwarfs (dark curves) and giants (light curves) from Wainscoat et al. (1992) have been added. Again, the shading indicates the number of objects per bin. [See the electronic edition of the Journal for a color version of this figure.]
TABLE 4

Summary of the Proper-Motion Sources

\begin{tabular}{|c|c|c|c|}
\hline SPM Flag & Catalog Name & $\begin{array}{l}\text { Number } \\
\text { of Sources }\end{array}$ & $\begin{array}{l}\text { Percentage } \\
\text { of DR1 }\end{array}$ \\
\hline ․․․……….... & No proper motion & 13 & 0.05 \\
\hline $1 \ldots \ldots \ldots \ldots \ldots \ldots \ldots$ & Tycho-2 & 218 & 0.86 \\
\hline $2 \ldots \ldots \ldots \ldots \ldots \ldots \ldots \ldots \ldots \ldots \ldots \ldots \ldots \ldots$ & SSS & 7396 & 29.3 \\
\hline 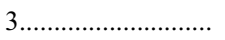 & Starnet 2.0 & 17465 & 69.1 \\
\hline 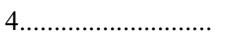 & 2MASS+GSC 1.2 & 182 & 0.72 \\
\hline
\end{tabular}

\subsection{Proper Motions}

The input catalog for the DR1 has been built on Tycho-2 and SSS, and both of these provide proper motions. When available, we also used proper motions from the Starnet 2.0 catalog, which is currently being compiled at the Astronomisches Rechen-Institut, Heidelberg. It is based on the Starnet catalog of the positions and proper motions (Röser 1996) of 4.3 million stars based on measurements from the Astrographic Catalog and GSC 1.2 (Morrison et al. 2001). The average rms error of proper motions in Starnet is $5 \mathrm{mas}^{-1}$, being mainly influenced by the accuracy of GSC 1.2. During the last decade new, highly accurate astrometric catalogs became available, namely, Tycho-2, UCAC2, and, for fainter stars, 2MASS. Starnet 2.0 combines Starnet with these three catalogs in a rigorous, weighted least-squares adjustment to derive mean positions and proper motions. Weights for the individual positions in the catalogs have been attributed according to the accuracy of the position measurements in the sources: for the Astrographic Catalog weights were taken from Röser \& Høg (1993), for GSC 1.2 from Morrison et al. (2001), for Tycho-2 from Høg et al. (2000), for UCAC2 from Zacharias et al. (2004), and for 2MASS from Cutri et al. (2003). The mean errors of the proper motions given in Starnet 2.0 are calculated from the individual weights. They are not calculated from the dispersion of the residuals due to the low degree of freedom in each individual adjustment. The average mean error in the proper motions of Starnet 2.0 stars in this cata$\log$ is 2.6 mas $^{-1} r^{-1}$ in each coordinate.

Neither Starnet nor Tycho-2 or UCAC2 are complete with regard to stars with proper motions in the range between approximately 30 and $150 \mathrm{mas} \mathrm{yr}^{-1}$. This is mainly due to the fact that the epoch difference between the Astrographic Catalog and modern catalogs is almost $100 \mathrm{yr}$. Therefore, the identifications of these moderately high proper motion stars is uncertain. To overcome this difficulty for Starnet 2.0, an auxiliary catalog has been constructed from the difference between 2MASS and GSC 1.2, with an epoch difference of about $20 \mathrm{yr}$. The average mean error in

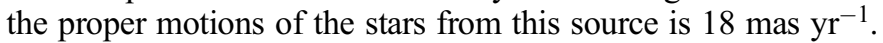
Proper motions are taken from this auxiliary catalog whenever no other proper motions could be found. A summary of the contribution of each proper motion source to DR1 is given in Table 4. The source of the proper motion is also flagged in the catalog (see Appendix A).

\subsection{Data Product and Data Access}

Due to the size of the catalog, RAVE DR1 is only accessible online. The catalog fields are nevertheless described in Tables 5-7. The catalog can be retrieved or queried from the RAVE collaboration Web site. ${ }^{35}$

As the catalog is large and will grow in size as further releases are made public, query tools are provided on this Web site. Users can access the catalog using different techniques, either with a

\footnotetext{
${ }^{35}$ See http://www.rave-survey.org.
} 
TABLE 5

Description of the RAVE Catalog

\begin{tabular}{|c|c|c|c|}
\hline Field Number & Name & NULL Value & Description \\
\hline .................. & OBJECTID & $\ldots$ & Internal identifier \\
\hline 2 & RA (deg) & $\ldots$ & Right ascension (J2000.0) \\
\hline 3 & $\mathrm{DE}(\mathrm{deg})$ & $\ldots$ & Declination (J2000.0) \\
\hline 4 & Glon (deg) & $\ldots$ & Galactic longitude \\
\hline 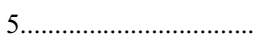 & Glat (deg) & $\ldots$ & Galactic latitude \\
\hline 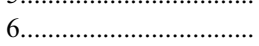 & $\mathrm{RV}\left(\mathrm{km} \mathrm{s}^{-1}\right)$ & $\cdots$ & Radial velocity \\
\hline 7 & $\operatorname{eRV}\left(\mathrm{km} \mathrm{s}^{-1}\right)$ & $\ldots$ & Internal radial velocity error \\
\hline 8 & pmRA $\left(\right.$ mas $\left.\mathrm{yr}^{-1}\right)$ & 9999.9 & Proper motion, right ascension \\
\hline 9 & epmRA $\left(\operatorname{mas} \mathrm{yr}^{-1}\right)$ & 9999.9 & Proper-motion error, right ascension \\
\hline 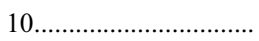 & $\operatorname{pmDE}\left(\operatorname{mas~} \mathrm{yr}^{-1}\right)$ & 9999.9 & Proper motion, declination \\
\hline $11 \ldots \ldots \ldots \ldots \ldots \ldots \ldots \ldots \ldots \ldots$ & epmDE $\left({\left.\operatorname{mas~} \mathrm{yr}^{-1}\right)}^{-1}\right.$ & 9999.9 & Proper-motion error, declination \\
\hline 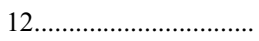 & Spm & $\ldots$ & Source proper motion (see $\S 6.3$ ) \\
\hline 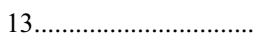 & Imag (mag) & $\ldots$ & Input catalog $I$ magnitude \\
\hline $14 \ldots \ldots \ldots \ldots \ldots \ldots \ldots \ldots . .$. & Obsdate (yyyymmdd) & $\ldots$ & Date of observation \\
\hline 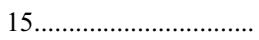 & FieldName & $\ldots$ & RAVE observations field \\
\hline $16 \ldots \ldots \ldots \ldots \ldots \ldots \ldots$ & FiberNumber & $\ldots$ & Fiber number on plate \\
\hline 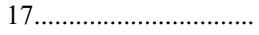 & CorrelationCoeff & $\ldots$ & Tonry-Davis correlation coefficient $R$ \\
\hline 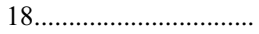 & PeakHeight & $\ldots$ & Height of the correlation function peak \\
\hline 19 & PeakWidth $\left(\mathrm{km} \mathrm{s}^{-1}\right)$ & $\ldots$ & Width of the correlation function peak \\
\hline 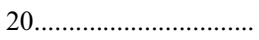 & CorrectionRV $\left(\mathrm{km} \mathrm{s}^{-1}\right)$ & $\ldots$ & Radial velocity correction applied \\
\hline 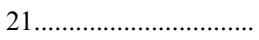 & SkyRV $\left(\mathrm{km} \mathrm{s}^{-1}\right)$ & $\ldots$ & Sky radial velocity in fiber \\
\hline 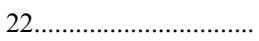 & SkyeRV $\left(\mathrm{km} \mathrm{s}^{-1}\right)$ & $\ldots$ & Sky radial velocity error \\
\hline 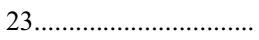 & SkyCorrelation & $\ldots$ & Tonry-Davis correlation coefficient for sky spectra \\
\hline 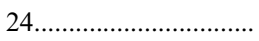 & PlateNumber & $\ldots$ & Physical plate number \\
\hline 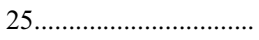 & SNRatio & $\ldots$ & Signal-to-noise ratio \\
\hline 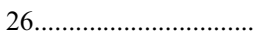 & BT (mag) & 99.99 & $B_{\mathrm{T}}$ magnitude from Tycho-2 \\
\hline 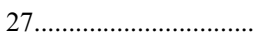 & eBT (mag) & 99.99 & $B_{\mathrm{T}}$-magnitude error from Tycho-2 \\
\hline 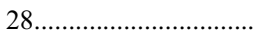 & VT (mag) & 99.99 & $V_{\mathrm{T}}$ magnitude from Tycho-2 \\
\hline 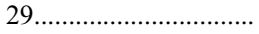 & eVT (mag) & 99.99 & $V_{\mathrm{T}}$-magnitude error from Tycho-2 \\
\hline 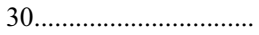 & USNOID & XXX & USNO-B identifier \\
\hline $31 \ldots \ldots \ldots \ldots \ldots \ldots \ldots \ldots \ldots \ldots$ & DisUSNO (mas) & 99.999 & Distance to USNO source \\
\hline 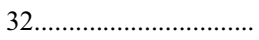 & $\mathrm{B} 1$ (mag) & 99.99 & USNO-B $B 1$ magnitude \\
\hline 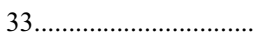 & $\mathrm{R} 1$ (mag) & 99.99 & USNO-B $R 1$ magnitude \\
\hline $34 \ldots \ldots \ldots \ldots \ldots \ldots \ldots \ldots \ldots \ldots .$. & B2 (mag) & 99.99 & USNO-B $B 2$ magnitude \\
\hline $35 \ldots \ldots \ldots \ldots \ldots \ldots \ldots \ldots \ldots \ldots \ldots \ldots \ldots \ldots \ldots$ & R2 (mag) & 99.99 & USNO-B $R 2$ magnitude \\
\hline 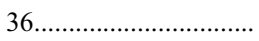 & IUSNO (mag) & 99.99 & USNO-B I magnitude \\
\hline 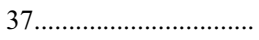 & XidQualityFLAGUSNO & $\mathrm{X}$ & Cross-identification quality FLAG USNO-B (see Table 6) \\
\hline 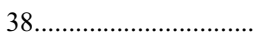 & DENISID & XXX & DENIS identifier \\
\hline 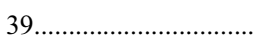 & DisDENIS (mas) & 99.999 & Distance to DENIS source \\
\hline 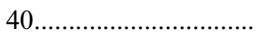 & IDENIS (mag) & 99.999 & DENIS I magnitude \\
\hline $41 \ldots \ldots \ldots \ldots \ldots \ldots \ldots \ldots \ldots \ldots$ & eIDENIS (mag) & 99.999 & DENIS I-magnitude error \\
\hline $42 \ldots \ldots \ldots \ldots-\cdots \cdots \cdots \cdots \cdots \cdots$ & JDENIS (mag) & 99.999 & DENIS $J$ magnitude \\
\hline 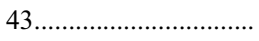 & eJDENIS (mag) & 99.999 & DENIS $J$-magnitude error \\
\hline 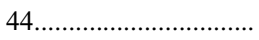 & KDENIS (mag) & 99.999 & DENIS $K$ magnitude \\
\hline 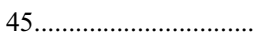 & eKDENIS (mag) & 99.999 & DENIS $K$-magnitude error \\
\hline 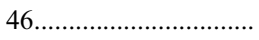 & XidQualityFLAGDENIS & $\mathrm{X}$ & Cross-identification quality FLAG DENIS (see Table 6) \\
\hline 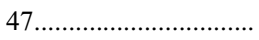 & TWOMASSID & XXX & 2MASS identifier \\
\hline 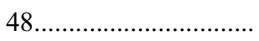 & Dis2MASS (mas) & 99.999 & Distance to 2MASS source \\
\hline 49 & J2MASS (mag) & 99.999 & 2MASS $J$ magnitude \\
\hline 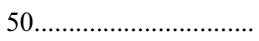 & eJ2MASS (mag) & 99.999 & 2MASS $J$-magnitude error \\
\hline 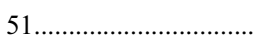 & H2MASS (mag) & 99.999 & 2MASS $H$ magnitude \\
\hline $52 \ldots \ldots \ldots \ldots \ldots \ldots \ldots \ldots \ldots \ldots \ldots \ldots \ldots \ldots \ldots \ldots \ldots$ & eH2MASS (mag) & 99.999 & 2MASS $H$-magnitude error \\
\hline 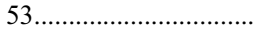 & K2MASS (mag) & 99.999 & 2MASS $K$ magnitude \\
\hline 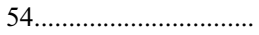 & eK2MASS (mag) & 99.999 & 2MASS $K$-magnitude error \\
\hline 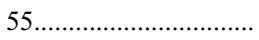 & TWOMASSphotFLAG & XXX & 2MASS photometry flag \\
\hline 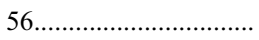 & XidQualityFLAG2MASS & $\mathrm{X}$ & Cross-identification quality flag 2MASS (see Table 6) \\
\hline 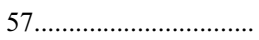 & ZeroPointQualityFLAG & $\ldots$ & Zero-point quality flag (see Table 1 ) \\
\hline 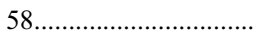 & VarFLAG & $\ldots$ & Variability flag, “*” if in GCVS2.0 \\
\hline 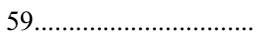 & SpectraQualityFLAG & $\ldots$ & Spectra quality flag (see Table 7) \\
\hline
\end{tabular}


TABLE 6

Summary of the Cross-identification Flag

\begin{tabular}{|c|c|}
\hline Flag Value & Description \\
\hline A........ & One association within $1^{\prime \prime}$ \\
\hline B.... & Two associations within $1^{\prime \prime}$ \\
\hline ........... & More than two associations within $1^{\prime \prime}$ \\
\hline (n................. & Nearest neighbor more than $2^{\prime \prime}$ away \\
\hline $\mathrm{X} \ldots \ldots \ldots$ & No association found \\
\hline
\end{tabular}

standard query interface or by field, using their position on the sky (this access mode is provided via a clickable map). For DR1 only tabular data are provided, and VOtable ${ }^{36}$ formatted outputs are offered for ease of use with the Virtual Observatory (VO) tools. For the subsequent releases, spectra will be made available, requiring the inclusion of more VO formats (enabling a proper description of a spectrum).

In the field query mode, links to the Aladin Sky Atlas (Bonnarel et al. 2000) are generated, providing more information in addition to VO tools to exploit the data. However, this link to Aladin is not provided for the standard catalog query, as the area on the sky is not restricted. The first data release catalog, as well as subsequent releases, will also be electronically available at the CDS in the VizieR database (Ochsenbein et al. 2000).

Again, DR1 includes RVs and associated errors from the RAVE survey, described in detail above. Cross matching with standard astrometric and photometric catalogs is provided for ease of use. Stellar parameters are not part of this release, as the firstyear spectra are contaminated by second-order light, which means that very detailed analysis is required to extract meaningful values.

\section{CONCLUSIONS}

This first data release presents RVs for 24,748 individual stars in the range $9 \lesssim I_{\text {DENIS }} \lesssim 12.5$, obtained from spectra in the infrared calcium-triplet region at a median resolution of 7500 . The total sky coverage of this catalog is $\sim 4760 \mathrm{deg}^{2}$. We demonstrated that the RVs are not affected by any trend in color or $\mathrm{S} / \mathrm{N}$ using both external data and RAVE repeat observations. The resulting variance for each set of validation data is consistent with our estimated errors.

DR1 does not include information about chemical abundances and other atmospheric parameters, for the reasons described above.

${ }^{36}$ VOtable is the standard format for tabular data in the VO.
The quality of the currently acquired spectra is good enough for derivation of $T_{\text {eff }}$, gravity, $[\mathrm{M} / \mathrm{H}],[\alpha / \mathrm{Fe}], V_{\text {rot }} \sin i$, and microturbulence (Fiorucci \& Munari 2005), and we expect to include chemical and atmospheric data in subsequent data releases.

RAVE is planned to observe until 2010 and will acquire up to $1,000,000$ spectra. Incremental releases, containing RVs and stellar parameters as well as spectra, are now planned on an approximately yearly basis, providing an unprecedented sample of stellar kinematics and chemical abundances in the range of magnitudes probing scales between the very local (Hipparcos-based) RV surveys (GCS; Famaey et al. 2005) and the more distant SDSS-II SEGUE and surveys with AAOmega, therefore completing our picture of the Milky Way.

We are indebted to the referee, Sydney van den Bergh, whose timely and justified comments helped us improve the clarity of the manuscript. M. S., A. S., and C. B. acknowledge financial support from the German Research Foundation. M. S. acknowledges financial support from the David and Lucile Packard Foundation. T. Z. acknowledges financial support from the Slovenian Research Agency. A. S. is supported by the Alexander von Humboldt Foundation. K. C. F., Q. A. P., R. C., J. B.-H., B. K. G., and M. W. are supported by an Australian Research Council Grant for the RAVE project. G. M. S. is funded by a Particle Physics and Astronomy Research Council Ph.D. Studentship. R. F. G. W. acknowledges seed money from the School of Arts and Sciences at Johns Hopkins University (JHU), plus NSF grant AST 05-08996. O. B. acknowledges financial support from the CNRS/INSU/PNG. E. K. G. is supported by the Swiss National Science Foundation under grants 200021-101924 and 200020-106260. A. H. and M. C. S. acknowledge financial support from NOVA and the Netherlands Organisation for Scientific Research. J. F. N. and J. P.'s participation in RAVE is supported by Canada's NSERC through a Special Research Opportunities grant. H. E. and M. S. acknowledge support by the Federal Ministry for Education and Research under FKZ 05AE2EE1/4 and 01AK804A. J. F. acknowledges support through grants from the W. M. Keck Foundation and the Gordon and Betty Moore Foundation to establish a program in data-intensive science at JHU.

The role of the Anglo-Australian Observatory in providing resources for the first 9 months of observation is gratefully acknowledged.

This research has made use of the VizieR catalog access tool, CDS, Strasbourg, France. Data verification is partially based

TABLE 7

Spectra Quality Flag Summary Table

\begin{tabular}{|c|c|}
\hline Flag Value & Description \\
\hline a........ & Asymmetric $\mathrm{Ca}$ lines \\
\hline 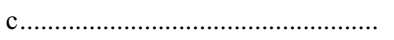 & Cosmic ray resulting in asymmetric correlation function \\
\hline е & Emission-line spectra \\
\hline $\mathrm{n}$ & Noise-dominated spectra, broad correlation function \\
\hline 1 & No lines visible, either strong noise or misplaced fiber \\
\hline $\mathrm{W}$ & Weak lines, radial velocity can be unreliable \\
\hline $\mathrm{g}$ & Strong ghost affecting the wavelength interval used for radial velocity calculation \\
\hline $\mathrm{t}$ & Bad template fit \\
\hline $\mathrm{s}$ & Strong residual sky emission \\
\hline $\mathrm{cc}$ & Bad continuum \\
\hline $\mathrm{r}$ & Red part of the spectra shows problems, noisy \\
\hline b & Blue part of the spectra shows problems, noisy \\
\hline $\mathrm{p}$ & Possible binary/double-lined \\
\hline
\end{tabular}

Note.-The values can be combined to give a more accurate description of the spectra. 
TABLE 8

List of RAVE Targets Observed with the ELODIE Spectrograph

\begin{tabular}{|c|c|c|c|c|c|c|c|c|c|}
\hline \multirow[b]{2}{*}{ IDENTIFIER } & \multicolumn{2}{|c|}{ RAVE } & \multicolumn{2}{|c|}{ ELODIE } & \multirow[b]{2}{*}{$\begin{array}{l}\text { Rad. Vel. DiFf. } \\
\left(\mathrm{km} \mathrm{s}^{-1}\right)\end{array}$} & \multirow[b]{2}{*}{$\begin{array}{c}V_{\mathrm{T}} / I \\
(\mathrm{mag})\end{array}$} & \multirow[b]{2}{*}{$\begin{array}{l}I_{\text {DENIS }} \\
(\mathrm{mag})\end{array}$} & \multirow[b]{2}{*}{$\begin{array}{r}J-H \\
(\mathrm{mag})\end{array}$} & \multirow[b]{2}{*}{$\begin{array}{r}H-K \\
(\mathrm{mag})\end{array}$} \\
\hline & $\begin{array}{l}\text { Rad. Vel. } \\
\left(\mathrm{km} \mathrm{s}^{-1}\right)\end{array}$ & $\begin{array}{l}\text { Rad. Vel. Error } \\
\quad\left(\mathrm{km} \mathrm{s}^{-1}\right)\end{array}$ & $\begin{array}{l}\text { Rad. Vel. } \\
\left(\mathrm{km} \mathrm{s}^{-1}\right)\end{array}$ & $\begin{array}{l}\text { Rad. Vel. Error } \\
\quad\left(\mathrm{km} \mathrm{s}^{-1}\right)\end{array}$ & & & & & \\
\hline T5027_00352_1........... & -7.9 & 2.2 & -8.9 & 1.0 & 1.0 & 10.43 & 9.03 & 0.57 & 0.16 \\
\hline T5027_00578_1........... & -32.0 & 1.5 & -32.3 & 1.0 & 0.3 & 10.58 & 9.55 & 0.34 & 0.14 \\
\hline T5027_00389_1........... & 39.9 & 2.0 & 39.0 & 1.0 & 0.9 & 11.18 & 9.74 & 0.68 & 0.17 \\
\hline T5027_00374_1........... & -47.5 & 1.7 & -43.7 & 1.0 & 0.2 & 11.42 & 10.14 & 0.66 & 0.17 \\
\hline T5031_00478_1 ${ }^{*} \ldots \ldots \ldots$ & -22.4 & 2.3 & -42.4 & 1.0 & 20.0 & 11.26 & $\ldots$ & 0.31 & 0.10 \\
\hline C1430054-094720...... & 92.7 & 2.1 & 91.9 & 1.0 & -0.2 & 11.47 & 11.85 & 0.62 & 0.11 \\
\hline C1429286-091608...... & -19.2 & 1.2 & -19.2 & 1.0 & 0.0 & 10.06 & 10.49 & 0.55 & 0.12 \\
\hline
\end{tabular}

NotES.-This table presents the RAVE observations as well as the ELODIE measurements. DENIS and 2MASS photometry are also reported. The RV errors for

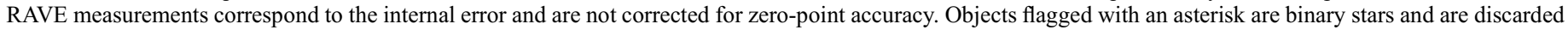
from the analysis. The $V_{\mathrm{T}} / I$ column contains the Tycho- $2 V_{\mathrm{T}}$ magnitudes for Tycho- 2 objects and the SSS $I$ magnitude for other targets.

on observations taken at the Observatoire de Haute Provence, France, operated by the French CNRS.

\section{APPENDIX A}

\section{CATALOG DESCRIPTION}

In Tables 5-7 we present the different columns that are provided in the first data release.

\section{APPENDIX B}

\section{EXTERNAL DATA}

In Tables 8-10 we present the calibration measurements obtained for the RAVE survey during the first year of operation. Those calibration data are divided according to their source (instrument or catalog). A complete description of those data is given in $\S 5.6$.

TABLE 9

2.3 m Observations of RAVE Targets

\begin{tabular}{|c|c|c|c|c|c|c|c|c|c|}
\hline \multirow[b]{2}{*}{ IDENTIFIER } & \multicolumn{2}{|c|}{ RAVE } & \multirow[b]{2}{*}{$\begin{array}{c}2.3 \mathrm{~m} \mathrm{RAD} \text { VEL. } \\
\left(\mathrm{km} \mathrm{s}^{-1}\right)\end{array}$} & \multirow[b]{2}{*}{$\begin{array}{l}\text { Rad. Vel. DifF. } \\
\left(\mathrm{km} \mathrm{s}^{-1}\right)\end{array}$} & \multirow[b]{2}{*}{$2.3 \mathrm{~m} \mathrm{R}$-V $\mathrm{V}$ ALUE } & \multirow[b]{2}{*}{$\begin{array}{c}V_{\mathrm{T}} / I \\
(\mathrm{mag})\end{array}$} & \multirow[b]{2}{*}{$\begin{array}{l}I_{\text {DENIS }} \\
(\mathrm{mag})\end{array}$} & \multirow[b]{2}{*}{$\begin{array}{l}J-H \\
(\mathrm{mag})\end{array}$} & \multirow[b]{2}{*}{$\begin{array}{r}H-K \\
(\mathrm{mag})\end{array}$} \\
\hline & $\begin{array}{l}\text { Rad. Vel. } \\
\left(\mathrm{km} \mathrm{s}^{-1}\right)\end{array}$ & $\begin{array}{l}\text { Rad. Vel. Error } \\
\quad\left(\mathrm{km} \mathrm{s}^{-1}\right)\end{array}$ & & & & & & & \\
\hline C1032220-225303 ............ & 103.2 & 2.4 & 103.6 & -0.4 & 43.4 & 11.82 & 12.43 & 0.30 & 0.01 \\
\hline C1032220-225303 ............ & 103.8 & 3.4 & 103.6 & 0.2 & 43.4 & 11.82 & 12.43 & 0.30 & 0.01 \\
\hline C1032264-241144 ............ & 52.4 & 2.1 & 54.3 & -1.9 & 75.9 & 11.85 & 12.20 & 0.32 & 0.08 \\
\hline C1032264-241144 ............ & 47.9 & 1.5 & 54.3 & -6.4 & 75.9 & 11.85 & 12.20 & 0.32 & 0.08 \\
\hline C1033394-215304_........... & 118.8 & 2.7 & 120.4 & -1.6 & 78.2 & 11.96 & 12.31 & 0.50 & 0.09 \\
\hline $\mathrm{C} 1033426-214025^{*} \ldots \ldots \ldots$ & 63.2 & 1.8 & 5.3 & 57.9 & 90.4 & 11.53 & 12.33 & 0.26 & 0.07 \\
\hline C1033426-214025* .......... & 48.2 & 2.3 & 5.3 & 42.9 & 90.4 & 11.53 & 12.33 & 0.26 & 0.07 \\
\hline C1033528-220832 _........... & 34.3 & 2.3 & 25.2 & 9.1 & 74.8 & 11.99 & 12.33 & 0.22 & 0.10 \\
\hline T6073_00197_1 ................... & 40.7 & 2.1 & 38.1 & 2.6 & 124.2 & 11.53 & 9.77 & 0.72 & 0.20 \\
\hline T6073_00197_1 ................... & 40.0 & 0.6 & 38.1 & 1.9 & 124.2 & 11.53 & 9.77 & 0.72 & 0.20 \\
\hline T6074_00342_1 .................. & -30.3 & 2.1 & -22.5 & -7.8 & 106.8 & 11.35 & 9.37 & 0.44 & 0.11 \\
\hline T6074_00342_1 .................. & -16.6 & 1.1 & -22.5 & 5.9 & 106.8 & 11.35 & 9.37 & 0.44 & 0.11 \\
\hline T6620_00749_1* ................ & -24.4 & 1.9 & 13.6 & -38.0 & 75.2 & 11.77 & 11.10 & 0.26 & 0.09 \\
\hline T6620_00749_1* ............... & -22.4 & 0.9 & 13.6 & -36.0 & 75.2 & 11.77 & 11.10 & 0.26 & 0.09 \\
\hline T6620_00941_1 .................. & 86.7 & 2.4 & 87.8 & -1.1 & 132.7 & 11.35 & 10.18 & 0.52 & 0.11 \\
\hline T6624_00025_1 ................... & 38.3 & 4.3 & 45.4 & -7.1 & 101.7 & 10.71 & 9.80 & 0.52 & 0.17 \\
\hline T6624_00025_1 ................... & 38.6 & 0.5 & 45.4 & -6.8 & 101.7 & 10.71 & 9.80 & 0.52 & 0.17 \\
\hline T6624_01181_1 .................. & 22.7 & 1.8 & 24.2 & -1.5 & 90.3 & 10.89 & 10.35 & 0.19 & 0.09 \\
\hline T6624_01181_1 ................... & 23.2 & 0.7 & 24.2 & -1.0 & 90.3 & 10.89 & 10.35 & 0.19 & 0.09 \\
\hline T6637_00126_1 ................... & 27.6 & 2.0 & 31.6 & -4.0 & 101.8 & 10.55 & 10.67 & 0.38 & 0.07 \\
\hline T6637_00126_1 .................. & 26.8 & 0.8 & 31.7 & -4.8 & 101.8 & 10.55 & 10.67 & 0.38 & 0.07 \\
\hline C1025107-255418_........... & 317.1 & 1.7 & 315.2 & 1.9 & 116.4 & 11.58 & 11.54 & 0.54 & 0.09 \\
\hline C1025107-255418............ & 317.2 & 1.0 & 315.2 & 2.0 & 116.4 & 11.58 & 11.54 & 0.54 & 0.09 \\
\hline C1025310-260312* ......... & 27.0 & 1.7 & -1.8 & 28.8 & 105.2 & 11.89 & 11.91 & 0.32 & 0.07 \\
\hline C1025310-260312* ......... & 1.3 & 2.1 & -1.8 & 3.1 & 105.2 & 11.89 & 11.91 & 0.32 & 0.07 \\
\hline C1026269-255018........... & 18.6 & 2.6 & 15.6 & 3.0 & 65.3 & 11.82 & 11.88 & 0.30 & 0.08 \\
\hline C1026269-255018............ & 16.5 & 1.8 & 15.6 & 0.9 & 65.3 & 11.82 & 11.88 & 0.30 & 0.08 \\
\hline T6623_00942_1 ................. & 16.7 & 1.8 & 13.2 & 3.5 & 93.4 & 10.06 & 9.42 & 0.36 & 0.15 \\
\hline T6623_00942_1 .................. & 13.9 & 0.9 & 13.2 & 0.7 & 93.4 & 10.06 & 9.42 & 0.36 & 0.15 \\
\hline T6627_01266_1* ............... & -3.0 & 2.6 & 36.2 & -39.2 & 78.9 & 10.87 & 10.26 & 0.17 & 0.11 \\
\hline T9317_01217_1 ................... & 7.0 & 2.0 & 11.1 & -4.1 & 78.6 & 10.71 & 9.91 & 0.22 & 0.06 \\
\hline T9459_00225_1 .................. & -6.0 & 1.1 & -7.2 & 1.2 & 103.4 & 10.66 & 10.16 & 0.27 & 0.06 \\
\hline T9459_00391_1 ................. & 5.5 & 1.8 & 7.2 & -1.7 & 73.3 & 11.22 & 10.10 & 0.63 & 0.09 \\
\hline
\end{tabular}


TABLE 9 - Continued

\begin{tabular}{|c|c|c|c|c|c|c|c|c|c|}
\hline \multirow[b]{2}{*}{ IDENTIFIER } & \multicolumn{2}{|c|}{ RAVE } & \multirow[b]{2}{*}{$\begin{array}{c}2.3 \text { m RAD. VEL. } \\
\left(\mathrm{km} \mathrm{s}^{-1}\right)\end{array}$} & \multirow[b]{2}{*}{$\begin{array}{l}\text { RaD. VeL. DIFF. } \\
\quad\left(\mathrm{km} \mathrm{s}^{-1}\right)\end{array}$} & \multirow[b]{2}{*}{$2.3 \mathrm{~m} R$-V Alue } & \multirow[b]{2}{*}{$\begin{array}{c}V_{\mathrm{T}} / I \\
(\mathrm{mag})\end{array}$} & \multirow[b]{2}{*}{$\begin{array}{l}I_{\text {DENIS }} \\
(\mathrm{mag})\end{array}$} & \multirow[b]{2}{*}{$\begin{array}{l}J-H \\
(\mathrm{mag})\end{array}$} & \multirow[b]{2}{*}{$\begin{array}{r}H-K \\
(\mathrm{mag})\end{array}$} \\
\hline & $\begin{array}{l}\text { Rad. Vel. } \\
\left(\mathrm{km} \mathrm{s}^{-1}\right)\end{array}$ & $\begin{array}{l}\text { Rad. Vel. Error } \\
\quad\left(\mathrm{km} \mathrm{s}^{-1}\right)\end{array}$ & & & & & & & \\
\hline T9459_00433_1* ............... & -17.9 & 2.3 & -30.7 & 12.8 & 53.0 & 10.24 & 9.93 & 0.16 & 0.02 \\
\hline T9459_00433_1* ............... & -22.0 & 2.1 & -30.7 & 8.7 & 53.0 & 10.24 & 9.93 & 0.16 & 0.02 \\
\hline T9459_01509_1 ..................... & 45.0 & 1.6 & 45.1 & -0.1 & 72.5 & 11.84 & 10.45 & 0.62 & 0.13 \\
\hline T9462_00133_1 .................. & -3.7 & 1.9 & -4.5 & 0.8 & 76.5 & 11.34 & 10.30 & 0.43 & 0.15 \\
\hline T9462_01174_1 ................... & -17.4 & 1.5 & -16.4 & -1.0 & 123.1 & 11.86 & 10.14 & 0.61 & 0.24 \\
\hline T9463_00658_1 ................... & 46.7 & 1.1 & 43.9 & 2.8 & 91.1 & 10.76 & & 0.30 & 0.10 \\
\hline T9463_01298_1 ................. & 35.6 & 1.9 & 36.3 & -0.7 & 75.6 & 11.42 & 10.50 & 0.37 & 0.05 \\
\hline T9467_00768_1 ................... & 71.8 & 1.6 & 74.5 & -2.7 & 111.4 & 11.57 & 9.79 & 0.71 & 0.21 \\
\hline T9318_00046_1 .................... & -0.5 & 1.3 & 1.1 & -1.6 & 81.3 & 11.80 & $\ldots$ & 0.60 & 0.09 \\
\hline T9318_00362_1 .................. & 15.1 & 1.6 & 15.8 & -0.7 & 92.3 & 10.64 & $\ldots$ & 0.17 & 0.09 \\
\hline T9319_00355_1 ................... & 2.0 & 1.7 & 3.5 & -1.5 & 96.9 & 10.74 & 9.63 & 0.47 & 0.12 \\
\hline T9458_01749_1 .................... & 38.2 & 1.0 & 38.3 & -0.1 & 117.1 & 11.52 & $\ldots$ & 0.75 & 0.20 \\
\hline T9458_02394_1 .................. & 114.4 & 1.6 & 116.8 & -2.4 & 106.3 & 11.61 & 10.23 & 0.73 & 0.11 \\
\hline T9459_00973_1 .................. & 85.4 & 1.3 & 86.4 & -1.0 & 94.1 & 10.62 & 9.68 & 0.53 & 0.06 \\
\hline 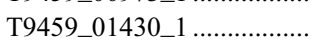 & -3.7 & 1.4 & -1.0 & -2.7 & 95.5 & 10.94 & 10.23 & 0.27 & 0.06 \\
\hline T9459_01430_1 .................. & -3.2 & 1.8 & -1.0 & -2.1 & 95.5 & 10.94 & 10.23 & 0.27 & 0.06 \\
\hline T9459_01822_1 ................... & 24.1 & 1.6 & 27.0 & -2.9 & 91.9 & 11.19 & 9.85 & 0.62 & 0.15 \\
\hline T9460_00265_1 ................... & -0.8 & 1.7 & 0.0 & -0.8 & 92.5 & 12.17 & 10.57 & 0.56 & 0.14 \\
\hline T9460_00353_1 ................... & -45.4 & 1.4 & -42.2 & -3.2 & 85.9 & 11.11 & 9.57 & 0.75 & 0.20 \\
\hline T9462_00226_1 .................. & -23.3 & 3.0 & -25.3 & 2.0 & 68.4 & 11.35 & 10.74 & 0.26 & 0.08 \\
\hline T9462_02202_1 .................. & 27.3 & 1.5 & 23.7 & 3.6 & 102.2 & 10.35 & 9.85 & 0.29 & 0.06 \\
\hline T9463_01633_1 .................. & 16.0 & 3.8 & 16.4 & -0.4 & 74.2 & 12.33 & 11.47 & 0.39 & 0.04 \\
\hline T9464_00658_1 ..................... & 1.3 & 1.0 & -0.7 & 2.0 & 80.7 & 10.82 & 10.09 & 0.22 & 0.07 \\
\hline T9464_00658_1 ................... & 0.4 & 1.5 & -0.7 & 1.1 & 80.7 & 10.82 & 10.09 & 0.22 & 0.07 \\
\hline T9464_00865_1 .................. & 8.3 & 1.5 & 3.2 & 5.1 & 67.2 & 10.76 & 9.96 & 0.33 & 0.15 \\
\hline T9467_00478_1 .................. & 23.1 & 6.6 & 22.4 & 0.7 & 98.9 & 11.80 & 10.24 & 0.67 & 0.22 \\
\hline T9317_01143_1 ............................ & 13.5 & 2.8 & 19.7 & -6.2 & 100.6 & 11.69 & 10.48 & 0.55 & 0.14 \\
\hline T9317_01143_1 .................. & 17.5 & 1.6 & 19.7 & -2.2 & 100.6 & 11.69 & 10.48 & 0.55 & 0.14 \\
\hline T9458_00843_1 ................... & 9.7 & 1.2 & 8.6 & 1.1 & 87.8 & 10.27 & 9.50 & 0.35 & 0.11 \\
\hline T9458_00934_1 .................. & -41.9 & 1.3 & -41.0 & -0.9 & 112.2 & 12.10 & 10.97 & 0.59 & 0.11 \\
\hline T9460_01761_1 .................... & 31.1 & 1.1 & 34.6 & -3.5 & 80.0 & 11.03 & 10.34 & 0.22 & 0.09 \\
\hline T9462_01690_1 .................. & 0.3 & 2.7 & 2.2 & -1.9 & 111.6 & 11.35 & 9.83 & 0.58 & 0.16 \\
\hline T9317_00415_1 .................... & 22.3 & 2.4 & 20.3 & 2.0 & 116.1 & 11.43 & 10.34 & 0.48 & 0.13 \\
\hline T9458_02020_1 .................. & 23.2 & 1.9 & 17.0 & 6.2 & 58.5 & 9.49 & 9.28 & -0.04 & 0.12 \\
\hline T9460_00219_1 ................... & 75.3 & 1.2 & 75.2 & 0.1 & 157.2 & 11.71 & 10.17 & 0.59 & 0.16 \\
\hline T9462_01219_1* ............... & 57.5 & 10.4 & 30.4 & 27.1 & 88.9 & 9.91 & 9.54 & 0.10 & 0.08 \\
\hline T9464_00050_1 .................. & 28.9 & 0.9 & 25.6 & 3.3 & 100.4 & 10.69 & 10.15 & 0.20 & 0.06 \\
\hline C1519196-191359............ & -407.3 & 2.3 & -409.0 & 1.7 & 76.8 & 11.32 & 12.12 & 0.66 & 0.14 \\
\hline C1508217-085010 .................... & -289.8 & 3.0 & -287.8 & -2.0 & 72.7 & 11.29 & 11.91 & 0.49 & 0.07 \\
\hline C1536201-144228 ............. & -329.2 & 2.8 & -330.4 & 1.2 & 46.4 & 11.82 & 12.42 & 0.49 & 0.16 \\
\hline T7274_00734_1 ................... & 407.9 & 1.5 & 407.8 & 0.1 & 108.2 & 10.06 & $\ldots$ & 0.46 & 0.07 \\
\hline C1905210-751503 ............. & -153.1 & 3.6 & -148.9 & -4.2 & 79.6 & 11.69 & 12.00 & 0.64 & 0.14 \\
\hline T8395_01513_1 ................... & -296.3 & 2.0 & -295.2 & -1.1 & 140.4 & 11.30 & 9.99 & 0.51 & 0.05 \\
\hline T6671_00389_1 ................... & 0.7 & 2.0 & -5.0 & 5.7 & 79.0 & 10.80 & 10.14 & 0.26 & 0.10 \\
\hline 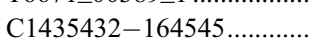 & -15.1 & 3.7 & -17.2 & 2.1 & 40.4 & 11.51 & 12.16 & 0.57 & 0.10 \\
\hline C1254296-164722 ............ & 71.7 & 2.2 & 74.3 & -2.6 & 44.0 & 10.82 & $\ldots$ & 0.38 & 0.08 \\
\hline C1532041-135910 ….......... & 44.2 & 1.4 & 36.8 & 7.4 & 43.5 & 11.81 & 12.09 & 0.45 & 0.09 \\
\hline C2030129-660620 ............. & 91.6 & 2.3 & 91.9 & -0.3 & 63.6 & 11.54 & 12.09 & 0.37 & 0.08 \\
\hline T7270_00796_1 ......................... & 151.4 & 1.5 & 152.4 & -1.0 & 81.1 & 11.88 & $\ldots$ & 0.44 & 0.08 \\
\hline T7524_00065_1 ................... & 353.2 & 1.2 & 353.8 & -0.6 & 130.1 & 11.37 & $\ldots$ & 0.55 & 0.14 \\
\hline 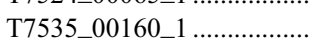 & 356.6 & 1.4 & 358.8 & -2.2 & 99.7 & 11.43 & 10.22 & 0.56 & 0.08 \\
\hline T9527_00088_1 .................. & -102.2 & 2.1 & -89.6 & -12.6 & 89.9 & 11.99 & 10.57 & 0.62 & 0.13 \\
\hline $\mathrm{C} 0314269-375257 \ldots \ldots \ldots \ldots$ & 69.3 & 1.8 & 72.9 & -3.6 & 83.8 & 11.97 & 12.38 & 0.48 & 0.13 \\
\hline $\mathrm{C} 2118490-174605 \ldots \ldots \ldots \ldots$ & -125.9 & 3.1 & -133.2 & 7.3 & 67.7 & 11.54 & 12.37 & 0.66 & 0.15 \\
\hline C2234046-564051 ............ & -12.0 & 2.0 & -15.2 & 3.2 & 77.8 & 11.88 & 12.05 & 0.51 & 0.12 \\
\hline C2330284-410842 ............. & 51.6 & 2.0 & 48.8 & 2.8 & 92.6 & 11.36 & 11.51 & 0.46 & 0.07 \\
\hline T7006_01317_1 .................... & 82.6 & 2.8 & 81.5 & 1.1 & 77.3 & 11.68 & $\ldots$ & 0.39 & 0.03 \\
\hline
\end{tabular}

Notes.-This table lists the RAVE targets observed with the $2.3 \mathrm{~m}$ long-slit spectrograph at Siding Spring. RAVE measurements, as well as DENIS $I$ magnitude, RV difference, and 2MASS colors, are also reported. Stars marked with asterisks are variable or binary objects and are discarded from the analysis. The estimated RV error for all $2.3 \mathrm{~m}$ data is $1.5 \mathrm{~km} \mathrm{~s}^{-1}$. 
TABLE 10

List of RAVE Targets in the Geneva-Copenhagen Survey

\begin{tabular}{|c|c|c|c|c|c|c|c|c|c|}
\hline \multirow[b]{2}{*}{ IDENTIFIER } & \multicolumn{2}{|c|}{ RAVE } & \multicolumn{2}{|c|}{ GENEVA } & \multirow[b]{2}{*}{$\begin{array}{l}\text { RAD. VEL. DIFF. } \\
\left(\mathrm{km} \mathrm{s}^{-1}\right)\end{array}$} & \multirow[b]{2}{*}{$\begin{array}{c}V_{\mathrm{T}} / I \\
(\mathrm{mag})\end{array}$} & \multirow[b]{2}{*}{$\begin{array}{r}I_{\text {DENIS }} \\
(\mathrm{mag})\end{array}$} & \multirow[b]{2}{*}{$\begin{array}{l}J-H \\
(\mathrm{mag})\end{array}$} & \multirow[b]{2}{*}{$\begin{array}{r}H-K \\
(\mathrm{mag})\end{array}$} \\
\hline & $\begin{array}{l}\text { Rad. Vel. } \\
\left(\mathrm{km} \mathrm{s}^{-1}\right)\end{array}$ & $\begin{array}{l}\text { Rad. Vel. Error } \\
\qquad\left(\mathrm{km} \mathrm{s}^{-1}\right)\end{array}$ & $\begin{array}{l}\text { Rad. Vel. } \\
\left(\mathrm{km} \mathrm{s}^{-1}\right)\end{array}$ & $\begin{array}{l}\text { Rad. Vel. Error } \\
\quad\left(\mathrm{km} \mathrm{s}^{-1}\right)\end{array}$ & & & & & \\
\hline 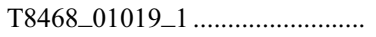 & 8.7 & 1.3 & 8.2 & 0.3 & 0.5 & 9.95 & $\ldots$ & 0.36 & 0.10 \\
\hline T6053_00177_1 ............................ & 57.9 & 1.4 & 55.4 & 0.7 & 2.5 & 11.56 & 10.39 & 0.59 & 0.10 \\
\hline 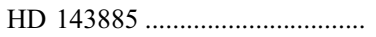 & 1.7 & 1.3 & -0.3 & 0.2 & 2.0 & 7.66 & 8.82 & 0.24 & 0.07 \\
\hline 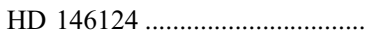 & 14.2 & 2.2 & 10.8 & 0.2 & 3.4 & 6.85 & $\cdots$ & 0.31 & 0.14 \\
\hline 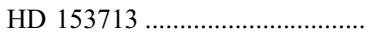 & -4.7 & 1.8 & -6.8 & 0.3 & 2.1 & 8.54 & 9.04 & 0.45 & 0.10 \\
\hline 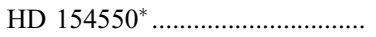 & -7.3 & 1.7 & -7.4 & $\ldots$ & 0.1 & 8.18 & 8.77 & 0.24 & 0.03 \\
\hline 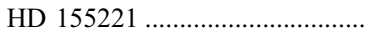 & -49.3 & 1.4 & -49.7 & 0.2 & 0.4 & 7.80 & 9.17 & 0.31 & 0.11 \\
\hline 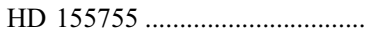 & -0.3 & 1.5 & -2.4 & 0.4 & 2.1 & 7.91 & $\ldots$ & 0.23 & 0.07 \\
\hline 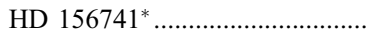 & -51.4 & 1.3 & -52.5 & 0.2 & 1.1 & 8.39 & 8.67 & 0.35 & 0.09 \\
\hline 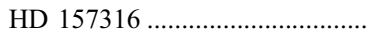 & -1.9 & 1.9 & -1.3 & 0.9 & -0.6 & 6.26 & 8.80 & 0.19 & 0.05 \\
\hline 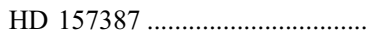 & 24.5 & 1.1 & 24.8 & 0.3 & -0.3 & 7.25 & 7.61 & 0.21 & 0.06 \\
\hline 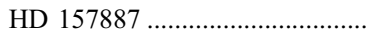 & -20.7 & 1.2 & -20.7 & 0.2 & 0.0 & 7.67 & 8.44 & 0.29 & 0.08 \\
\hline T8454_00006_1 .......................... & 7.8 & 1.5 & 4.3 & 0.2 & 3.5 & 10.18 & $\ldots$ & 0.38 & 0.08 \\
\hline
\end{tabular}

Notes.- This table contains the Geneva-Copenhagen data together with RAVE measurements. DENIS and 2MASS photometry are reported for convenience. Objects flagged with asterisks are binary stars and are discarded from the analysis.

Abadi, M. G., Navarro, J. F., \& Steinmetz, M. 2006, MNRAS, 365, 747

Abadi, M. G., Navarro, J. F., Steinmetz, M., \& Eke, V. R. 2003, ApJ, 597, 21

Belokurov, V., et al. 2006, ApJ, 642, L137

Bessell, M. S. 1979, PASP, 91, 589 1986, PASP, 98, 1303

Blair, M., \& Gilmore, G. 1982, PASP, 94, 742

Bonnarel, F., et al. 2000, A\&AS, 143, 33

Brook, C., Gibson, B., Martel, H., \& Kawata, D. 2005, ApJ, 630, 298

Bullock, J. S., \& Johnston, K. V. 2005, ApJ, 635, 931

Colless, M., et al. 2001, MNRAS, 328, 1039

Cutri, R. M., et al. 2003, The 2MASS All-Sky Catalog of Point Sources (Pasadena: Caltech)

Dehnen, W. 2000, AJ, 119, 800

de Simone, R., Wu, X., \& Tremaine, S. 2004, MNRAS, 350, 627

Eggen, O. J., Lynden-Bell, D., \& Sandage, A. R. 1962, ApJ, 136, 748

Famaey, B., Jorissen, A., Luri, X., Mayor, M., Udry, S., Dejonghe, H., \& Turon, C. 2005, A\&A, 430, 165

Fiorucci, M., et al. 2005, BAAS, 37, 1367

Freeman, K. C., \& Bland-Hawthorn, J. 2002, ARA\&A, 40, 487

Gilmore, G., Wyse, R. F. G., \& Jones, J. B. 1995, AJ, 109, 1095

Gilmore, G., Wyse, R. F. G., \& Norris, J. E. 2002, ApJ, 574, L39

Governato, F., et al., 2004, ApJ, 607, 688

Hambly, N. C., Irwin, M. J., \& McGillivray, H. T. 2001a, MNRAS, 326, 1295

Hambly, N. C., et al. 2001b, MNRAS, 326, 1279

Helmi, A., Navarro, J. F., Nordström, B., Holmberg, J., Abadi, M. G., \& Steinmetz, M. 2006, MNRAS, 365, 1309

Høg, E., et al. 2000, A\&A, 355, L27

Ibata, R. A., Gilmore, G., \& Irwin, M. J. 1994, Nature, 370, 194

Ibata, R., Irwin, M., Lewis, G., Ferguson, A. M. N., \& Tanvir, N. 2001, Nature, 412, 49

Jones, D. H., et al. 2004, MNRAS, 355, 747

Katz, D., et al. 2004, MNRAS, 354, 1223

Kuijken, K., \& Gilmore, G. 1989a, MNRAS, 239, 571

. 1989b, MNRAS, 239, 605 1989c, MNRAS, 239, 651

Kurtz, M. J., Mink, D. J., Wyatt, W. F., Fabricant, D. G., Torres, G., Kriss, G. A., \& Tonry, J. L. 1992, in ASP Conf. Ser. 25, XCSAO: A Radial Velocity Package for the IRAF Environment, ed. D. M. Worrall, C. Biemesderfer, \& J. Barnes (San Francisco: ASP), 432

Lewis, I. J., et al. 2002, MNRAS, 333, 279

Meza, A., Navarro, J. F., Abadi, M. G., \& Steinmetz, M. 2005, MNRAS, 359, 93

Miszalski, B. 2006, MNRAS, submitted

Morrison, J. E., Röser, S., McLean, B., Bucciarelli, B., \& Lasker, B. 2001, AJ, 121,1752

Munari, U., ed. 2003, ASP Conf. Ser. 298, Gaia Spectroscopy: Science and Technology (San Francisco: ASP)

\section{REFERENCES}

Munari, U., Agnolin, P., \& Tomasella, A. 2001, Baltic Astron., 10, 613

Munari, U., Sordo, R., Castelli, F., \& Zwitter, T. 2005, A\&A, 442, 1127

Navarro, J. F., Helmi, A., \& Freeman, K. C. 2004, ApJ, 601, L43

Newberg, H. J., et al. 2003, BAAS, 35, 1385

Nordström, B., et al. 2004, A\&A, 418, 989

Ochsenbein, F., Bauer, P., \& Marcout, J. 2000, A\&AS, 143, 23

Parker, Q. A., \& Watson, F. G. 1990, A\&AS, 84, 455

Perryman, M. A. C., et al. 1997, The Hipparcos and Tycho Catalogues (ESA SP-1200; Noordwijk: ESA)

Piquard, S., Halbwachs, J.-L., Fabricius, C., Geckeler, R., Soubiran, C., \& Wicenec, A. 2001, A\&A, 373, 576

Quillen, A. C., \& Minchev, I. 2005, AJ, 130, 576

Robertson, B., Bullock, J. S., Font, A. S., Johnston, K. V., \& Hernquist, L. 2005, ApJ, 632, 872

Rodgers, A. W., Conroy, P., \& Bloxham, G. 1988, PASP, 100, 626

Röser, S. 1996, in IAU Symp. 172, Dynamics, Ephemerides, and Astrometry of the Solar System, ed. S. Ferraz-Mello, B. Morando, \& J.-E. Arlot (Dordrecht: Kluwer), 481

Röser, S., \& Høg, E. 1993, in TYCHO Reference Catalogue, ed. A. G. D. Philip, B. Hauck, \& A. R. Upgren (Schenectady: Davis), 137

Seabroke, G. M. 1879, MNRAS, 39, 450

-1887, MNRAS, 47, 93

. 1889, MNRAS, 50, 72

Sommer-Larsen, J., Gotz, M., \& Portinari, L. 2003, ApJ, 596, 47

Steinmetz, M., \& Navarro, J. F. 2002, NewA, 7, 155

Tolstoy, E., Venn, K. A., Shetrone, M., Primas, F., Hill, V., Kaufer, A., \& Szeifert, T. 2003, AJ, 125, 707

Tonry, J., \& Davis, M. 1979, AJ, 84, 1511

Unavane, M., Wyse, R. F. G., \& Gilmore, G. 1996, MNRAS, 278, 727

Vogel, H. C. 1873, Astron. Nachr., 82, 291

Wainscoat, R. J., Cohen, M., Volk, K., Walker, H. J., \& Schwartz, D. E. 1992, ApJS, 83, 111

Watson, F. G., Parker, Q. A., Bogatu, G., Farrell, T. J., Hingley, B. E., \& Miziarski, S. 2000, Proc. SPIE, 4008, 123

Wilkinson, M., et al. 2005, MNRAS, 359, 1306

Wyse, R. F. G., \& Gilmore, G. 1992, MNRAS, 257, 1 1995, AJ, 110, 2771

- 2006, in ASP Conf. Proc., Resolved Stellar Populations, ed. D. VallsGabaud \& M. Chavez, in press (astro-ph/0510025)

Yanny, B., et al. 2003, ApJ, 588, 824

Zacharias, N., Urban, S. E., Zacharias, M. I., Wycoff, G. L., Hall, D. M., Monet, D. G., \& Rafferty, T. J. 2004, AJ, 127, 3043

Zwitter, T. 2002, A\&A, 386, 748

Zwitter, T., Castelli, F., \& Munari, U. 2004, A\&A, 417, 1055

Zwitter, T., \& Munari, U. 2004, Rev. Mex. AA Ser. Conf., 21, 251 